\title{
Article \\ Cycloastragenol Inhibits Experimental Abdominal Aortic Aneurysm Progression
}

\author{
Leander Gaarde Melin 1,2,3 ${ }^{\mathbb{D}}$, Julie Husted Dall 1,2,3 ${ }^{\text {, Jes S. Lindholt }}{ }^{1,2}$, Lasse B. Steffensen ${ }^{3} \mathbb{D}$, \\ Hans Christian Beck ${ }^{1,4}{ }^{(D)}$, Sophie L. Elkrog ${ }^{3}$, Pernille D. Clausen ${ }^{3} \mathbb{D}$, Lars Melholt Rasmussen ${ }^{1,4}$ \\ and Jane Stubbe $1,3, * \mathbb{D}$
}

1 Centre for Individualized Medicine in Arterial Diseases (CIMA), Odense University Hospital (OUH), 5000 Odense, Denmark; leander.gaarde@rsyd.dk (L.G.M.); julie.husted.dall@regionh.dk (J.H.D.); jes.sanddal.lindholt@rsyd.dk (J.S.L.); hans.christian.beck@rsyd.dk (H.C.B.); lars.melholt.rasmussen@rsyd.dk (L.M.R.)

2 Department of Cardiothoracic and Vascular Surgery, Odense University Hospital, 5000 Odense, Denmark

3 Cardiovascular and Renal Research Unit, Institute for Molecular Medicine, University of Southern Denmark, 5000 Odense, Denmark; lsteffensen@health.sdu.dk (L.B.S.); soelk17@student.sdu.dk (S.L.E.); pecla17@student.sdu.dk (P.D.C.)

4 Department of Clinical Biochemistry and Pharmacology, Odense University Hospital, 5000 Odense, Denmark

* Correspondence: jstubbe@health.sdu.dk; Tel.: +45-6550-3709

check for updates

Citation: Melin, L.G.; Dall, J.H.; Lindholt, J.S.; Steffensen, L.B.; Beck, H.C.; Elkrog, S.L.; Clausen, P.D.; Rasmussen, L.M.; Stubbe, J. Cycloastragenol Inhibits Experimental Abdominal Aortic Aneurysm Progression. Biomedicines 2022, 10, 359. https://doi.org/ 10.3390/biomedicines10020359

Academic Editor: Elena Kaschina

Received: 23 December 2021

Accepted: 30 January 2022

Published: 2 February 2022

Publisher's Note: MDPI stays neutral with regard to jurisdictional claims in published maps and institutional affiliations.

Copyright: (C) 2022 by the authors. Licensee MDPI, Basel, Switzerland. This article is an open access article distributed under the terms and conditions of the Creative Commons Attribution (CC BY) license (https:// creativecommons.org/licenses/by/ $4.0 /)$.

\begin{abstract}
The pathogenesis of abdominal aortic aneurysm involves vascular inflammation and elastin degradation. Astragalus radix contains cycloastragenol, which is known to be anti-inflammatory and to protect against elastin degradation. We hypothesized that cycloastragenol supplementation inhibits abdominal aortic aneurysm progression. Abdominal aortic aneurysm was induced in male rats by intraluminal elastase infusion in the infrarenal aorta and treated daily with cycloastragenol (125 mg/kg/day). Aortic expansion was followed weekly by ultrasound for 28 days. Changes in aneurysmal wall composition were analyzed by mRNA levels, histology, zymography and explorative proteomic analyses. At day 28, mean aneurysm diameter was 37\% lower in the cycloastragenol group $(p<0.0001)$. In aneurysm cross sections, elastin content was insignificantly higher in the cycloastragenol group $(10.5 \% \pm 5.9 \%$ vs. $19.9 \% \pm 16.8 \%, p=0.20)$, with more preserved elastin lamellae structures $(p=0.0003)$ and without microcalcifications. Aneurysmal matrix metalloprotease2 activity was reduced by the treatment $(p=0.022)$. Messenger RNA levels of inflammatory- and anti-oxidative markers did not differ between groups. Explorative proteomic analysis showed no difference in protein levels when adjusting for multiple testing. Among proteins displaying nominal regulation were fibulin-5 ( $p=0.02)$, aquaporin-1 $(p=0.02)$ and prostacyclin synthase $(p=0.007)$. Cycloastragenol inhibits experimental abdominal aortic aneurysm progression. The suggested underlying mechanisms involve decreased matrix metalloprotease-2 activity and preservation of elastin and reduced calcification, thus, cycloastragenol could be considered for trial in abdominal aortic aneurysm patients.
\end{abstract}

Keywords: aortic aneurysm; pathogenesis; pharmacological therapy; experimental model; drug delivery

\section{Introduction}

Abdominal aortic aneurysm (AAA) is a localized enlargement of the aorta exceeding $3 \mathrm{~cm}$ in diameter and is potentially life-threatening [1]. Globally, AAA rupture is a major cause of mortality in elderly men, being responsible for the death of $1 \%$ of men above 65 years [1,2]. Today, patients with known AAAs are carefully monitored and offered surgical repair when the AAA possesses a diameter above $5-6 \mathrm{~cm}$. Thus, an urgent unmet clinical need of medical therapies for small AAAs exists, to prevent progressive dilatation, acute or elective surgical repair, rupture, and death [3-5]. 
Chronic inflammation, due to persistent infiltration of inflammatory cells into the aortic wall and degradation of elastin, seems to fundamentally characterize the pathology of AAAs [6]. Elastin in the medial layer of the aortic wall is degraded when the aneurysm is formed. This loss of elastin is partly compensated by the continuous formation of collagen and elastin by vascular smooth muscle cells along with expression of structural proteins such as tropoelastin and fibrillin 1, which is cross-linked by lysyl oxidase (LOX) and fibulin-5 to form elastic fibers [6-8]. This process is diminished by inflammation when elastin-degrading enzymes, such as matrix metalloproteases (MMPs), are released from macrophages, monocytes, neutrophils and activated vascular smooth muscle cells [6,7]. Especially the M1 proinflammatory macrophages, which produce proinflammatory cytokines and MMPs, augment AAA progression [9]. Moreover, reactive oxygen species (ROS) contribute to AAA expansion by enhancing MMP activity, which further degrades the extracellular matrix (ECM) and weakens the aneurysm wall [6,10-13].

A promising dietary supplement in the fight against AAA progression is cycloastragenol (CAG). It is a crystalline solid triterpenoid saponin compound and a hydrolyzed product of the main active ingredient in the Chinese herb Astragalus membranaceus. CAG has been used in traditional Chinese medicine for over 2000 years with no commonly known side effects [14]. Recent literature describes various anti-inflammatory effects of CAG in heart, vascular, liver and skin tissue including inhibition of lymphocyte activation. CAG also reduces MMP-2 and MMP-9 expression and activity, thereby preserving extracellular matrix (ECM) integrity [15-19]. CAG supplement has recently been proven to attenuate AAA expansion in mice using an elastase wrapping model and in angiotensin-II induced AAA in Apolipoprotein E (ApoE) $)^{-/-}$mice [19]. However, the model can be criticized for not producing human-like-AAA in contrast to the elastase perfusion model in rats, which uses porcine pancreatic elastase (PPE) applied intraluminally in the infrarenal aortic segment through laparotomy and atherectomy [20]. This imitates human AAA well, as it displays various pathological similarities, such as inflammation, elastin degradation, thrombus formation and calcification [21]. Thus, the growing base of evidence describing the anti-inflammatory properties of CAG illustrates its potential as a possible future medical treatment against AAA expansion in humans.

In this study, we hypothesize that CAG inhibits the progressive dilatation of AAA in the rat PPE aneurysm-model by its anti-inflammatory and anti-oxidative effects leading to reduced protease activity and, thereby, preserving elastin integrity.

\section{Materials and Methods}

\subsection{Study Design}

The rats were randomly allocated to either CAG treatment $(125 \mathrm{mg} / \mathrm{kg} /$ day $)$ or controls, starting on the first postoperative day, by an external investigator, not participating in any of the experimental procedures. Therefore, rats were housed independent of treatment, and daily caretakers were blinded to treatment.

\subsection{Outcomes}

The primary outcome was peak-systolic infrarenal aortic anterior to posterior innerto-inner diameter. Secondary explanatory outcome were: AAA wall content of elastin, its structure and LOX mRNA levels. Determine the effect of CAG on matrix-dependent MMP2, -9 and -12 mRNA levels and MMP activity. Measure mRNA levels of leukocyte marker CD45 mRNA, macrophage marker F4/80 mRNA and IL-6 and -10 mRNA levels together with the pro-inflammatory M1 macrophage marker iNOS, the antioxidative markers Nrf2 and HO-1 in the aneurysms. Furthermore, perform histological assessments of cluster of differentiation (CD)68 and CD206 and the presence of calcification in the aneurysm wall. Finally, identify potential new targets of CAG treatment in aneurysm wall by discovery proteomic analysis.

Potential harm outcomes were weight of liver, spleen, heart, and kidneys, as well as morphology of the inferior right liver lobe. 


\subsection{Sample Size Calculation}

We have, in previous experiments with the rat model, observed a mean diameter increase of $158.75 \% \pm 77.5 \mathrm{SD}$. To detect a $50 \%$ difference, which is considered clinically relevant, by a $t$-test using $5 \%$ significance level and $80 \%$ power, 24 rats are needed (12 in each group). This sample size estimation is conservative, as two-way repeated measure ANOVA tests were used to determine the correlation of the aortic diameters between the groups over time.

\subsection{Experimental Animals, Ethical Statement, Housing and Husbandry}

Male Sprague-Dawley rats purchased from Janvier Laboratories, Le Genest-Saint-Isle, France, were housed in cages of up to 4 rats per cage under twelve-hours light/dark cycle, room temperature of $20^{\circ} \mathrm{C}$, air humidity of $55 \%$ with free access to standard chow and tap water at the Biomedical Laboratory at the University of Southern Denmark. Rats were acclimatized for at least one week after delivery before entering the experimental protocol.

All animal experiments were conducted in accordance with a protocol ethically approved by the Danish Animal Experiments Inspectorate (license nr. 2016-15-0201-01046), and in accordance with arrive guidelines [22]. As females are generally protected against AAA formation $[1,2,23,24]$, we only used males in this study.

2.5. Induction of Abdominal Aortic Aneurysm by Perfusion of Pancreatic Porcine Elastase (PPE) in the Infrarenal Region of the Aorta

On the day of surgery, male Sprague-Dawley rats (260-435 g corresponding to age 7-10 weeks) were given $0.2 \mathrm{mg}$ temgesic (buprenorphine, Indivior, North Chesterfield, VA, USA) administered in $1 \mathrm{~g}$ of nut paste (Nutella) for pain management [25]. Then, the rats were anesthetized by a subcutaneous injection of a mixture of fentanyl $(236 \mu \mathrm{g} / \mathrm{kg})$, fluanisone (7.5 mg/kg, Skanderborg Apotek, Skanderborg, Denmark) and midazolam (3.75 mg/kg, Hameln Pharma, Hamelin, Germany) and underwent AAA induction by intraluminal PPE infusion of the infrarenal region by the procedure previously described by Shack et al. [26]. The only variation was that pancreatic porcine elastase concentration was increased to 12 units $/ \mathrm{mL}$ for $30 \mathrm{~min}$ and post-surgical pain management was provided with additional $0.2 \mathrm{mg}$ temgesic in nut paste (Nutella) [25]. The surgical procedure lasted between $60-70 \mathrm{~min}$. If needed rats were supplemented with $20 \%$ of the initial dose of anesthesia by subcutaneous injection during the operation. Representative pictures of the surgical steps and post-surgery day 28 are shown in Figure 1. All rats were observed post-surgery until they were fully awake from anesthesia. They were housed in individual cages in a heated cabinet until next morning, where treatment of the rats was initiated, and the rats were housed together throughout the rest of the experimental period. Rats were treated daily from day 1 post-surgery with CAG $(125 \mathrm{mg} / \mathrm{kg}$, Chengdu King-tiger Pharmchem. Tech. Co., Ltd., Chengdu, China, $\mathrm{n}=12$ ) or vehicle (deionized reverse osmosis water containing $0.05 \%$ methylcellulose $(w / v$, Merck), $2 \%$ tween $80(v / v$, Merck), $\mathrm{n}=12)$ by oral gavage using Soft Flex feeding tubes (Vetagree, Oslo, Norway) for 28 consecutive days; AAA expansion was monitored weekly by ultrasound measurements, as described below. Four rats were excluded; two vehicle controls died due to surgical and post-surgical complications and two did not consistently receive CAG due to difficulties with oral gavage.

\subsection{Ultrasound Measurement of Aneurysm Progression}

The abdominal aorta was video recorded from the renal artery to the bifurcation using ultrasound (LogiQ e ultrasound machine and a L10-22-RS transducer, GE Healthcare, Brøndby, Denmark) on the day of AAA induction, day 0 prior to surgery and, thereafter, on days 7, 14, 21 and 28 during treatment using $4 \%$ isoflurane inhalation anesthesia (SigmaAldrich, Søborg, Denmark). All ultrasound scans were performed by the same investigator, as our pilot study of 76 ultrasound recordings from 16 rats displayed a $6.9 \%$ variance between two investigators and stored for later analysis. Measurements of maximal vertical anterior to posterior diameter of the aorta spanning from internal edges during peak 
systolic blood pressure were performed blinded to the treatment group by two independent investigators using standard software on the LogiQ e Ultrasound machine. Inter-observer variation of the measured diameters was determined to be $3.7 \%$ based on the 76 pilot study ultrasound recordings. Values for the relative increase were obtained by adjusting to the diameter on day 0.
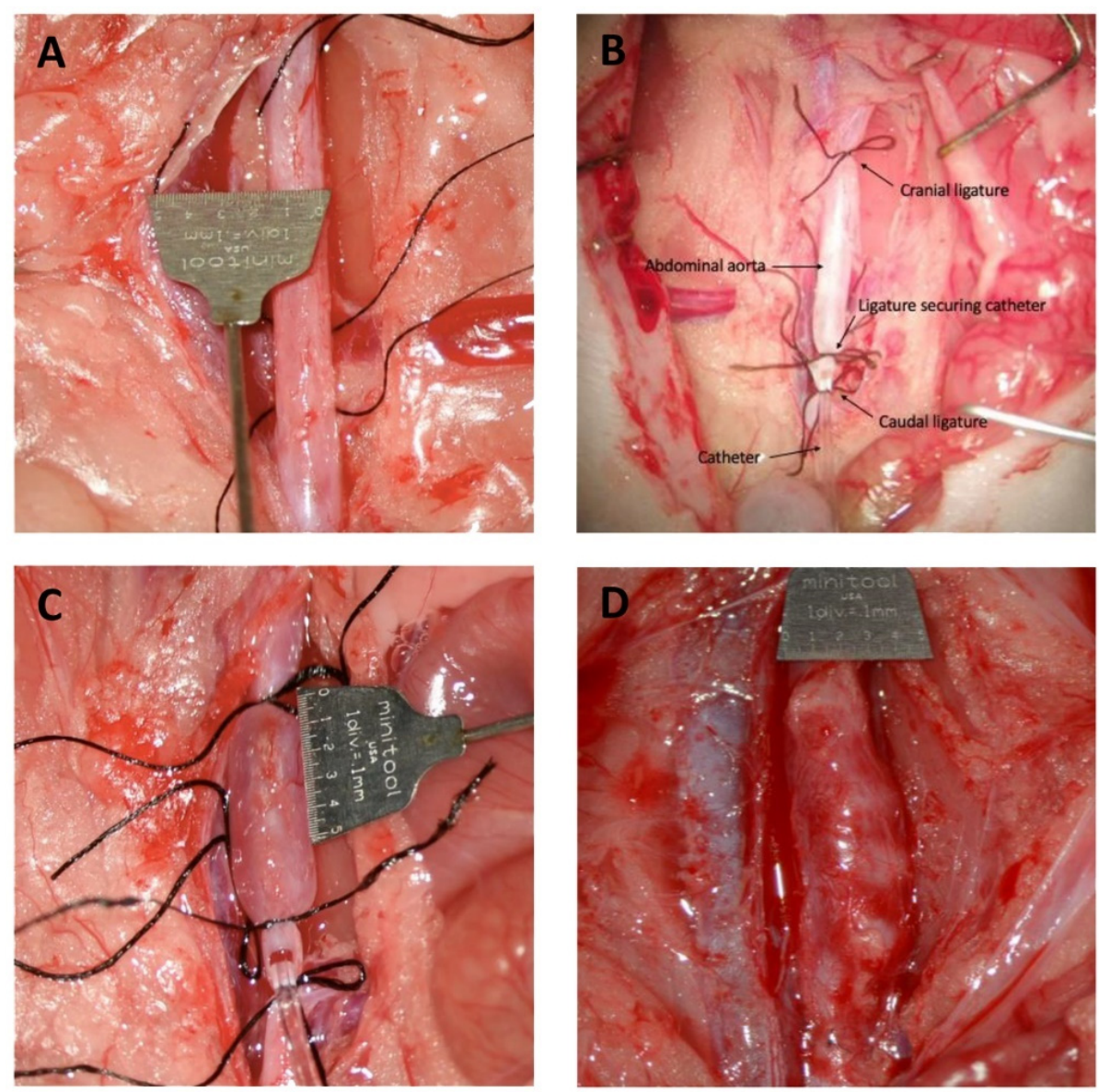

Figure 1. Induction of the surgical steps. (A) Isolated infrarenal aorta, (B) placing catheter in the infrarenal aorta, (C) intraluminal porcine pancreatic elastase infusion, (D) isolated AAA on day 28 post surgery.

\subsection{At Termination}

All rats were euthanized by exsanguination 28 days after AAA induction, resulting in 10 rats in each group. Liver, spleen, heart, and kidneys were collected and weighed. Subsequently, a specimen from the inferior right liver lobe was fixed as described below for morphological analysis.

The aneurysm specimens were isolated and divided into two pieces; the cranial piece was fixed in a 10\% normal formalin buffer (Hounisen Laboratorie udstyr A/S, Skanderborg, Denmark) over night at $4{ }^{\circ} \mathrm{C}$, then placed in phosphate-buffered saline (PBS) (Thermo Fisher, Slangerup, Denmark) with 0.05\% azide (Sigma-Adrich, Søborg, Denmark) and subsequently embedded in paraffin for morphological analysis. Two samples from the vehicle-group and three from the CAG-group, were unfortunately damaged in the embedding process. The caudal piece was immediately snap frozen in liquid nitrogen and kept at $-80{ }^{\circ} \mathrm{C}$ until RNA and protein isolation.

\subsection{Miller's Elastin and Calcium Von Kossa's Staining}

Five $\mu \mathrm{m}$ cross-sections of paraffin embedded aneurysm specimens were sectioned, deparaffinized and hydrated. For identification of elastin fibers, Miller's elastin stain kit 
(Atom Scientific, Hyde, UK) was used according to the manufacturer's instructions. In brief, sections were stained for $3 \mathrm{~h}$ in Miller's elastin stain, subsequently washed and counterstained with Van Gieson's stain.

Micro-calcium deposits in the aneurysm wall were detected by the silverplating kit according to Von Kossa's stain instructions (Merck, Søborg, Denmark). Calcium deposits were visualized using a 20-watt energy-saving lamp (Quantification method, described below). One additional sample from the vehicle group was damaged during the Von Kossa staining process.

\subsection{Immunohistochemistry}

Aneurysm cross-sections were deparaffinized and hydrated, followed by antigen retrieval by heating to $100{ }^{\circ} \mathrm{C}$ for $15 \mathrm{~min}$ in a citrate buffer (10 mM; pH 6, Merck, Søborg, Denmark) for CD206, MMP2or in a TEG-buffer (10 mM TrisBase; 0.5 mM EGTA; pH 9, Sigma Aldrich, Søborg, Denmark) for CD68.

Sections were subsequently blocked for endogenous peroxide activity using, respectively, a 3\% and a 1.5\% hydrogen peroxide (Merck, Søborg, Denmark) in tris-buffered saline (TBS, Sigma Aldrich, Søborg, Denmark) solution for, respectively, 10 and $30 \mathrm{~min}$. This was followed by one hour blocking in a 5\% milk/1× TBS/0.05\% Tween-20 (TBST) solution for CD68 and $\alpha$-actin 3\% BSA-TBST solution for CD206 and MMP2.

After washing in TBST, the aneurysm sections were incubated overnight at $4{ }^{\circ} \mathrm{C}$ with primary anti-CD68 (Abcam, Cambridge, UK) 1:500 and $\alpha$-actin (Sigma Aldrich, Søborg, Denmark) 1:500 in 5\% milk/TBST and anti-CD206 (Abcam, Cambridge, UK) 1:1000 and MMP2 (Abcam, Cambridge, UK) 1:500, both in 3\% BSA/TBST. The next day sections were washed in TBST and incubated with horseradish peroxidase (HRP) conjugated goat-anti-rabbit (DAKO, Glostrup, Denmark) 1:1000 or HRP conjugated anti mouse (DAKO, Glostrup, Denmark) 1:1000 in 5\% milk/TBST or 3\% BSA/TBST. Positive staining was visualized with 3,3'-Diaminobenzidine tetrahydrochloride hydrate (DAB, Merck, Søborg, Denmark) and sections were counterstained in Mayer's hematoxylin (Merck, Søborg, Denmark) and rinsed in tap water. As negative controls rabbit immunoglobulin IgG corresponding to the primary antibody concentration was applied (DAKO, Glostrup, Denmark). All staining was analyzed in an Olympus Bx51 microscope and micrographs were captured using an Olympus DP26 camera. After analysis, whole frame micrographs were adjusted for brightness and contrast using Photoshop (ver. 9, San Jose, CA, USA).

\subsection{Elastin Content Analysis and Immunohistochemical Cell Count}

For the assessment of elastin percentage in the medial layer, Image J software (ImageJ 1.53a Wayne Rasband, National Institutes of Health, Bethesda, MD, USA) was used. The external edge of the medial layer was defined as the transition site from disrupted or concentric rings of elastin to connective tissue in the adventitial layer. To quantify the percentage of elastin, the color threshold tool was used. For the scoring of aneurysmal wall disruption, each micrograph was divided into 8 areas, and each field was scored from 1-4, 4 being severe wall disruption and 1 minimal wall disruption. All assessments of elastin content were performed by two investigators blinded to treatment. The interobserver variation was $2.2 \%$ and the average score was used for statistical calculations.

Thereafter, elastin lamellae externa was used to identify the border between the medial layer and adventitial layer when identifying the adventitial area with CD68 and CD206 positive cell count per $\mathrm{mm}^{2}$. The total area of adventitial layer was divided by the number of positive labeled cells to determine positive cells per $\mathrm{mm}^{2}$. One investigator blinded to treatment determined numbers of positive CD68 and CD206 cells per adventitial area.

\subsection{Zymography}

Aneurysm samples were homogenized in protein extraction buffer $(0.3 \mathrm{M}$ sucrose; 25 mM Imidazole, 1 mM EDTA, pH 7.2 complete protease inhibitor cocktail 2 and 3, Sigma 
Aldrich, Søborg, Denmark). Samples were centrifuged for $10 \mathrm{~min}$ at $6000 \times g$ at $4{ }^{\circ} \mathrm{C}$. Protein concentration was determined by Bicinchoninic Acid Kit for Protein Determination (Sigma Aldrich, Søborg, Denmark) using bovine serum albumin as the standard. A total of $12 \mu \mathrm{g}$ protein samples and 1.25 $\mu \mathrm{L}$ recombinant MMP-2 (Sigma Aldrich, Søborg, Denmark) were mixed with an equal amount of $2 \times$ tris-glycine SDS sample buffer (Thermo Fischer) loaded onto a Novex zymogram gel containing 10\% gelatin (Thermo Fisher, Slangerup, Denmark) and proteins were separated by gel electrophoresis at $125 \mathrm{~V}$ for $90 \mathrm{~min}$. Proteins were then allowed to refold $30 \mathrm{~min}$ in renaturation buffer (Thermo Fisher, Slangerup, Denmark) followed by $24 \mathrm{~h}$ at $37^{\circ} \mathrm{C}$ in developing buffer (Thermo Fischer, Slangerup, Denmark). Finally, undigested proteins in the gel were stained with simple blue stain (Thermo Fisher) for $30 \mathrm{~min}$. White bands were inverted and quantified in Molecular Imager Image Lab (ChemiDoc WRS+, Biorad, Copenhagen, Denmark).

\subsection{Proteomic Analysis}

Preparation of AAA tissue for mass spectrometry was performed as previously described [27]. In brief, snap frozen tissue was homogenized in a lysis buffer, then denatured, alkylated, and digested with trypsin overnight. Tryptic peptides were purified on custom-made Poros R2/R3 (Thermo Fisher Scientific, Slangerup, Denmark) columns, and peptide concentration was normalized across samples. Samples (4 $\mathrm{g}$ tryptic peptides per sample) were randomly labelled with 10-plex tandem mass tags (TMT, Thermo Scientific, Waltham, MA, USA); mass tag 126 was a pool of all AAA samples and served as internal control. Proteome data are protein abundances relative to the internal control. Mixed peptide samples were high-pH fractionated and analyzed by nano-LC-MS/MS virtually, as previously described [28]. All Eclipse raw data files were processed and quantified using Proteome Discoverer version 2.4 (Thermo Scientific, Waltham, MA, USA) as previously described [28].

\subsection{Quantitative Polymerase Chain Reaction Measurements ( $q P C R$ )}

The methods of total RNA isolation, cDNA synthesis and qPCR quantification have been described previously by Wintmo et al. [29]. Addition of $1 \mu \mathrm{L}$ glycoblue (Thermo Fisher, Slangerup, Denmark) as a carrier for enhancement of RNA precipitation was the only modification. Primers used for determining mRNA levels are shown in Table 1.

Table 1. Primer sequences for qPCR analyses. The coefficient of correlation obtained for the standard curve expressed as $\mathrm{R}^{2}$-value is stated for each PCR product.

\begin{tabular}{|c|c|c|c|}
\hline Target & Forward Primer $\left(5^{\prime}-3^{\prime}\right)$ & Reverse Primer $\left(5^{\prime}-3^{\prime}\right)$ & $\mathbf{R}^{2}$-Value \\
\hline Lysyl oxidase (LOX) & ACCTGGTACCCGATCCCTAC & AGTCTCTGACATCCGCCCTA & 0.99 \\
\hline Inducible nitric oxidase synthase (iNOS) & AGGCAAGCCCTCACCTACTT & GATGGGAACTCTTCCAGCAC & 0.98 \\
\hline Mature macrophages (F4/80) & TTTTGGCTGCTCCTCTTCTG & TGGCATAAGCTGGACAAGTG & 0.98 \\
\hline Interleukin-6 (IL-6) & CAGAGTCATTCAGAGCAATAC & CTTTCAAGATGACTTGGATGG & 0.98 \\
\hline Interleukin-10 (IL-10) & TCTCCCCTGTGAGAATAAAA & TAGACACCTTTGTCTTGGAG & 0.96 \\
\hline Matrix Metalloprotease 2 (MMP-2) & GATCTTCTTCCTTCAAGGATCG & TACACGGCATCAATCTTTTC & 0.99 \\
\hline Matrix Metalloprotease 9 (MMP-9) & TACTTTGGAAACGCAAATGG & GTGTAGGATTCTACTGGG & 0.99 \\
\hline Matrix Metalloprotease 12 (MMP-12) & CAATATTGGAGGTACGATGTG & GTCATATTCCAATTGGTAGGC & 0.90 \\
\hline Cluster of differentation 45 (CD45) & GCTATAAAAGACCCCTTCAG & CATTAGGCAAATAGAGACACTG & 0.99 \\
\hline Heme oxygenase 1 (HO-1) & ACAGAAGAGGCTAAGACCG & CAGGCATCTCCTTCCATT & 0.99 \\
\hline Nuclear factor erythroid-2-related factor (Nrf2) & CCATTTGTAGATGACCATGAG & CTATTAAGACACTGTAACTCGG & 0.95 \\
\hline Ribsomal Protein L41 (RPL41) & TGGCGGAAGAAGAGAATGC & TGGACCTCTGCCTCATCTTT & 0.99 \\
\hline
\end{tabular}


Messenger RNA (mRNA) levels of genes of interest and five standards (10-fold dilutions) were run in duplicate using SYBR green (Biorad, Copenhagen, Denmark) as the detector system. RNase-free water and RNA samples without reverse transcriptase were used as negative controls. All samples were loaded on 96 Aria Max well plates (Agilent Technologies, Santa Clara, CA, USA), and the PCR amplification was done using three steps (initial $3 \mathrm{~min}$ at $95^{\circ} \mathrm{C}$, followed by 40 cycles: $95^{\circ} \mathrm{C} 20 \mathrm{~s} ; 60^{\circ} \mathrm{C} 20 \mathrm{~s}, 72{ }^{\circ} \mathrm{C} 15 \mathrm{~s}$ ) followed by a melting curve for the determination of PCR-product selectivity. RNA yield in one sample from each group was low; therefore, these samples were only included in RPL41, LOX, F4/80 and iNOS. Each mRNA expression level of the gene of interest was normalized to the complimentary expression level of the housekeeping gene ribosomal protein L41 (RPL41) that we first tested and did not change significantly between vehicleand CAG-treated aneurysms.

\subsection{Statistical Methods}

D'Angostino and Pearson test was used for normality testing. A two-way repeated measures ANOVA adjusted for weight at entry with a Greenhouse-Geisser correction, due to the violation of the assumption of sphericity (Mauchly's test), was used to analyze difference in relative aneurysm diameter between groups, calculated in SPSS (IBM SPSS Statistics, IBM Corporation, Endicott, NY, USA, 1989, 2020). Sidak's multiple comparison test was subsequently applied in Graphpad Prism (ver. 8, San Diego, CA, USA) for each time point.

For the secondary explanatory data, two-tailed unpaired Student's t-test was used to analyze normally distributed data. Values are presented as mean \pm standard deviation (SD). Welch's correction was used if F-test for variance was significant. A non-parametric Mann-Whitney test was used if data failed normality testing by the D'Angostino and Pearson test. Values are then presented as median \pm interquartile range (IQR). Chi-square test was used for categorial outcome variables.

Explorative proteomics data were analyzed by Student's t-test for each protein and subsequent false discovery rate (FDR) correction for multiple testing and GO enrichment analysis was performed using default settings of the DAVID Bioinformatics Resources [30,31]. The $p$-values $<0.05$ were considered significant.

\section{Results}

All rats tolerated daily treatment well, except for the two CAG rats excluded due to difficulties with the daily gavage. Treatment did, however, seem to affect liver, spleen, and heart to body weight ratios with a higher ratio among CAG treated rats (Supplementary Figure S1A-C), while kidney to body weight ratio was unaffected (Supplementary Figure S1D). Microscopic analysis of HE-stained liver lobes displayed no obvious differences as evaluated by investigators. As mentioned, a total of four rats were excluded causing an unintendedly higher non-significant mean initial body weight in the vehicle treated rats when compared to the CAG-treated group. Furthermore, there was a large variation in body weight within groups (vehicle: $351.8 \mathrm{~g} \pm 52.2 \mathrm{~g}$ vs. CAG: $332.0 \mathrm{~g} \pm 50.1 \mathrm{~g}, p=0.33, \mathrm{n}=10 / 10$ ). To make sure initial body weight did not influence AAA expansion, the statistical analysis was adjusted for body weight. Body weight increase during the experimental period was similar in both groups (Supplementary Figure S1E, $35.6 \% \pm 11.3 \%$ vs. $36.78 \% \pm 11.2 \% ; p=0.83, \mathrm{n}=10 / 10$ ).

\subsection{CAG Treatment Inhibited AAA Expansion}

The relative increase in aortic aneurysm diameter at the widest point during peak systolic blood pressure, adjusted for weight at entry, increased gradually in both groups during the experimental period of 28 days (Figure 2). CAG treatment led to significantly smaller aneurysms on days 7, 14, 21 and 28 compared to vehicle treatment (Figure 2). Aneurysm growth was most pronounced during the first 14 days after induction and reached maximal enlargement after 21 days with a mean relative increase of $124 \% \pm 10 \%$ and $88 \% \pm 10 \%$ for 
vehicle and CAG groups, respectively (Figure 2). No further change in aneurysm growth was observed at day 28 in either group.

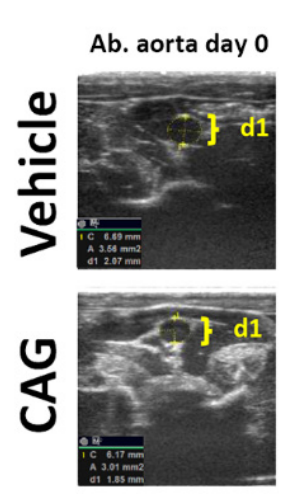

Site of AAA
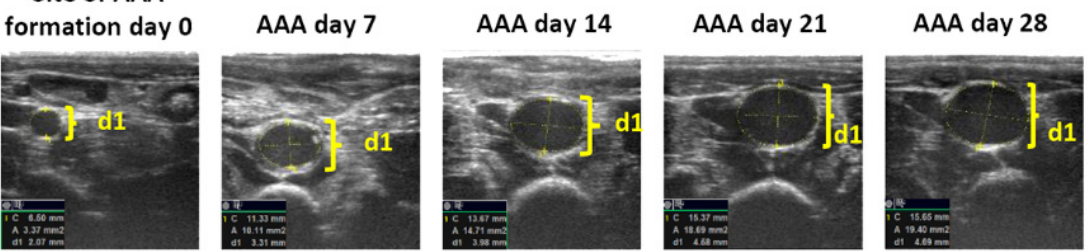

Ab. aorta day 28
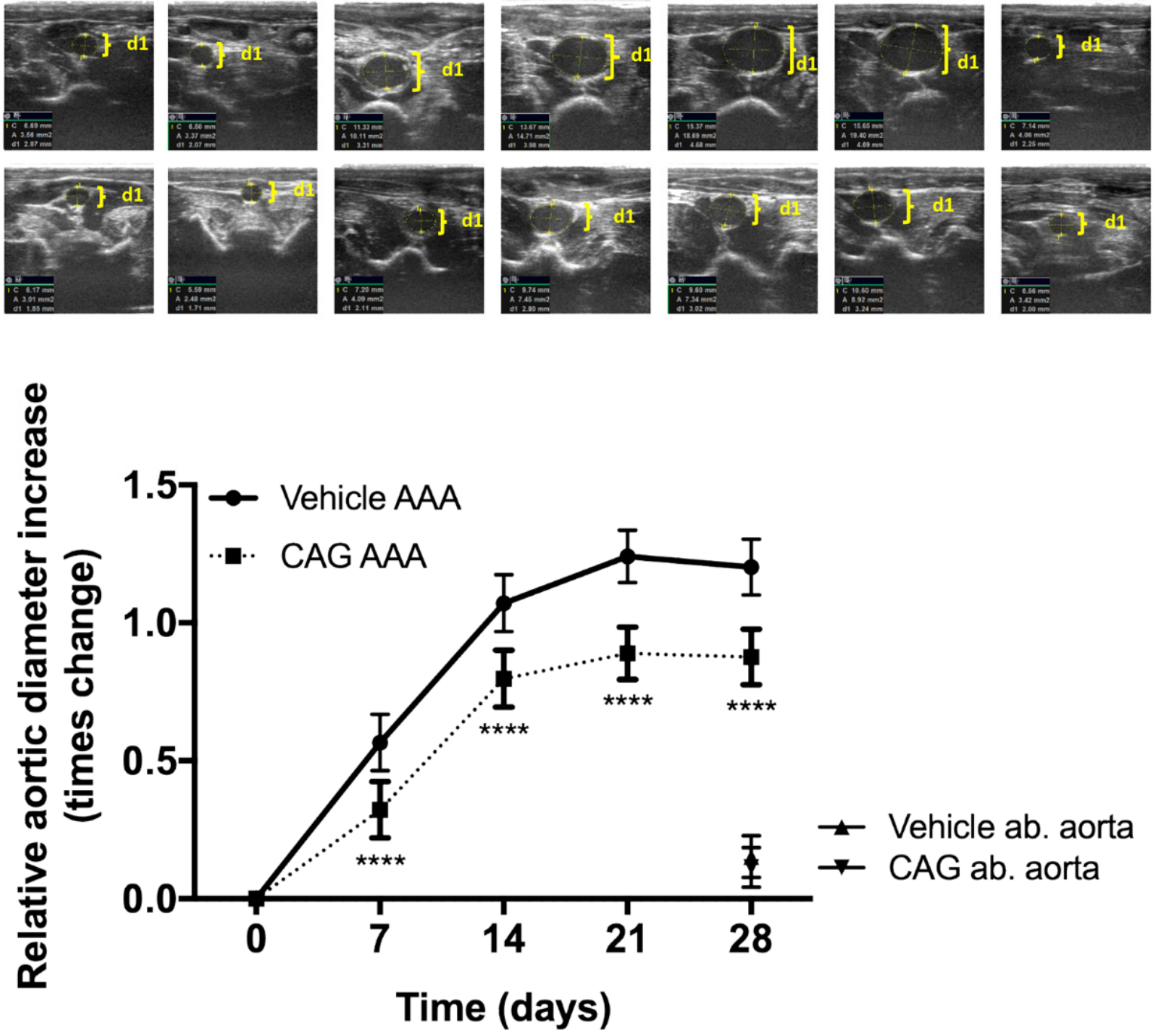

Figure 2. The effect of CAG on AAA growth. Upper panel shows representative ultrasound recordings at baseline and post-surgery days 7, 14, 21, 28 for both vehicle and CAG treated rats. The abdominal aorta (Ab. Aorta) just distal from the left renal artery was used as a reference point for developmental aortic expansion in the experimental period (day 0 and post-surgery day 28). D1: shows aortic diameter. Below, the relative increase in maximal aortic aneurysm diameter adjusted for weight at entry from day 0-28 measured by ultrasound in CAG treated group and vehicle treated group $(n=10 / 10)$. Values are mean \pm standard deviation. ${ }^{* * * *}$ indicates $p<0.0001$.

\subsection{CAG Treatment Affects Elastin Integrity}

Comparing cross sections of aneurysms at day 28 from both groups to unaffected abdominal aortas proximal to the aneurysm, revealed significant degradation and disruption of elastin lamellae in the medial layer (Figure 3A). Assessing the elastin content in the medial layer in both the vehicle and CAG treated AAA sections showed the mean percentage of elastin content was nearly doubled in the CAG treated group compared to the vehicle treated group, though not significantly (Figure $3 \mathrm{~B}, p=0.20$ ). Elastin degradation and disruption was not affected uniformly in the aneurysm cross sections. Scoring 8 areas on each cross-section showed that areas more prone to rupture (grade 4) were significantly more pronounced in the vehicle-treated group, while larger areas in the CAG-treated group 
were minimally affected, with large areas scoring grade 1 (Figure $3 C, p=0.0003$ ), indicating less wall thinning and destruction, thus, less potential for rupture. This protective effect on elastin was most likely not caused by augmented synthesis of elastin, as the mRNA levels of lysyl oxidase (LOX), an enzyme involved in elastin synthesis and cross-linking [7], was similar in both groups (Figure 3D, $p=0.57$ ).

A
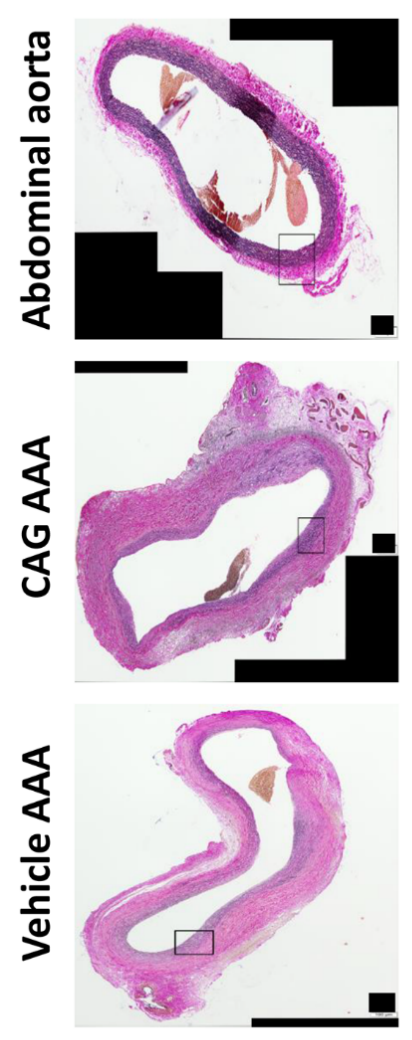

C
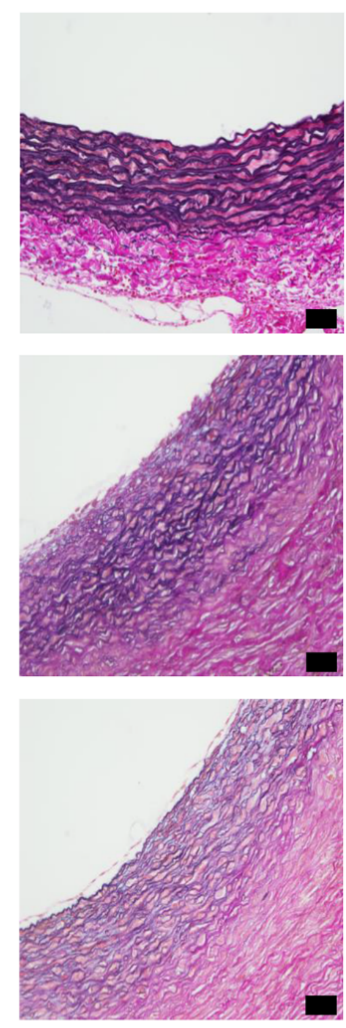

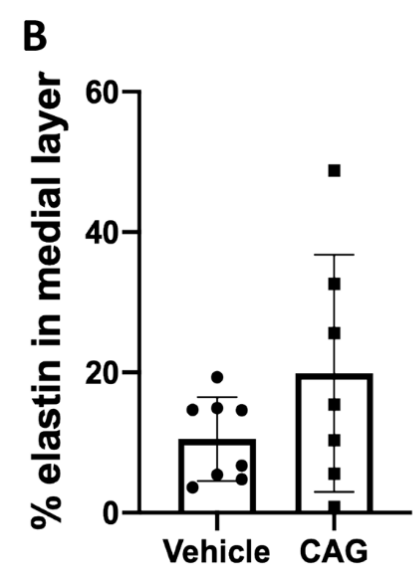

D

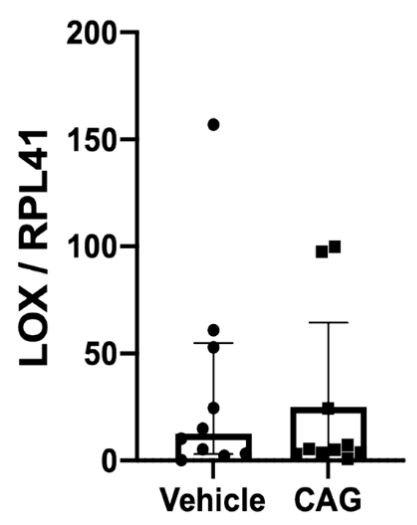

***

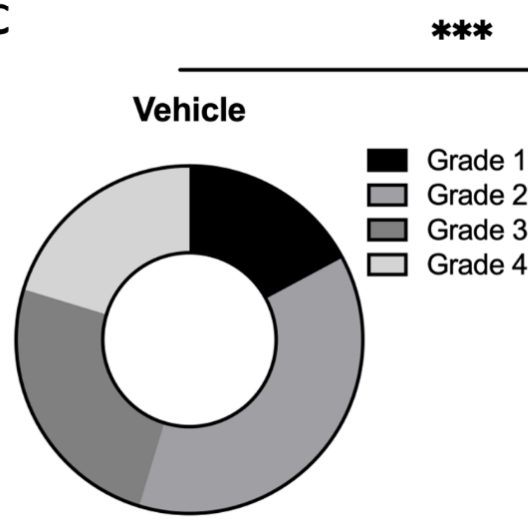

Total $=64$

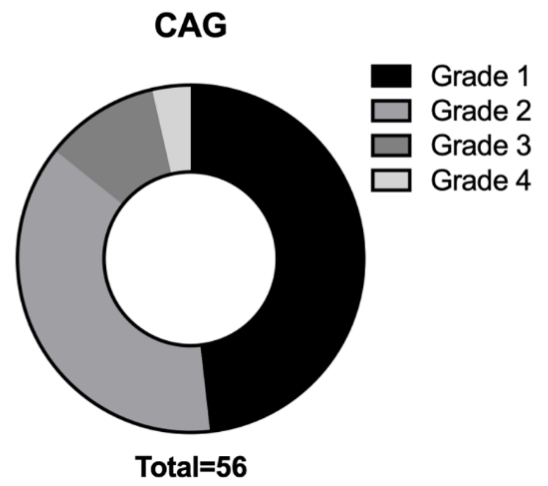

Figure 3. Elastin assessment in abdominal aortic aneurysms (AAA) (A) Representative micrographs of Miller's elastin stain (black) from abdominal aorta, vehicle AAA and CAG AAA at day 28 (enlargements correspond to black square on the left image). Scale bar (black box) in micrographs to the left: $100 \mu \mathrm{m}$ and micrographs to the right: $50 \mu \mathrm{m}$ (B) Percentage of elastin in medial layer in vehicle and CAG group AAA at day $28(n=8 / 7)$. (C) Scoring of aneurysmal wall elastin disruption in vehicle and CAG group 1-4, 4 being severe wall disruption and 1 minimal wall disruption ( $\mathrm{n}=8 / 7$; $p=0.0003)$. (D) Elastin related mRNA coding for LOX gene $(n=10 / 10)$ normalized to RPL41 mRNA levels. Values are median \pm inter quartile range. ${ }^{* * *}$ Indicates $p<0.001$. 
To investigate whether CAG protects against elastin degradation, mRNA levels of MMPs known to play a major role in AAA development [32] were determined. Neither mRNA levels of MMP-2 ( $p=0.22)$, MMP-9 $(p=0.24)$ nor MMP-12 $(p=0.60)$ were affected by CAG treatment (Figure 4A-C). In contrast, MMP-2 activity measured by zymography was significantly decreased in the CAG-treated group (Figure 4D), suggesting CAG treatment partly prevents elastin degradation and AAA growth by dampening MMP-2 activity. MMP2 was associated with a subset of vascular smooth muscle cells in the aneurysm wall, where weak labeling of MMP-2 was detected in the medial layer. There was no apparent difference between the two groups (Figure $4 \mathrm{E}$ ).
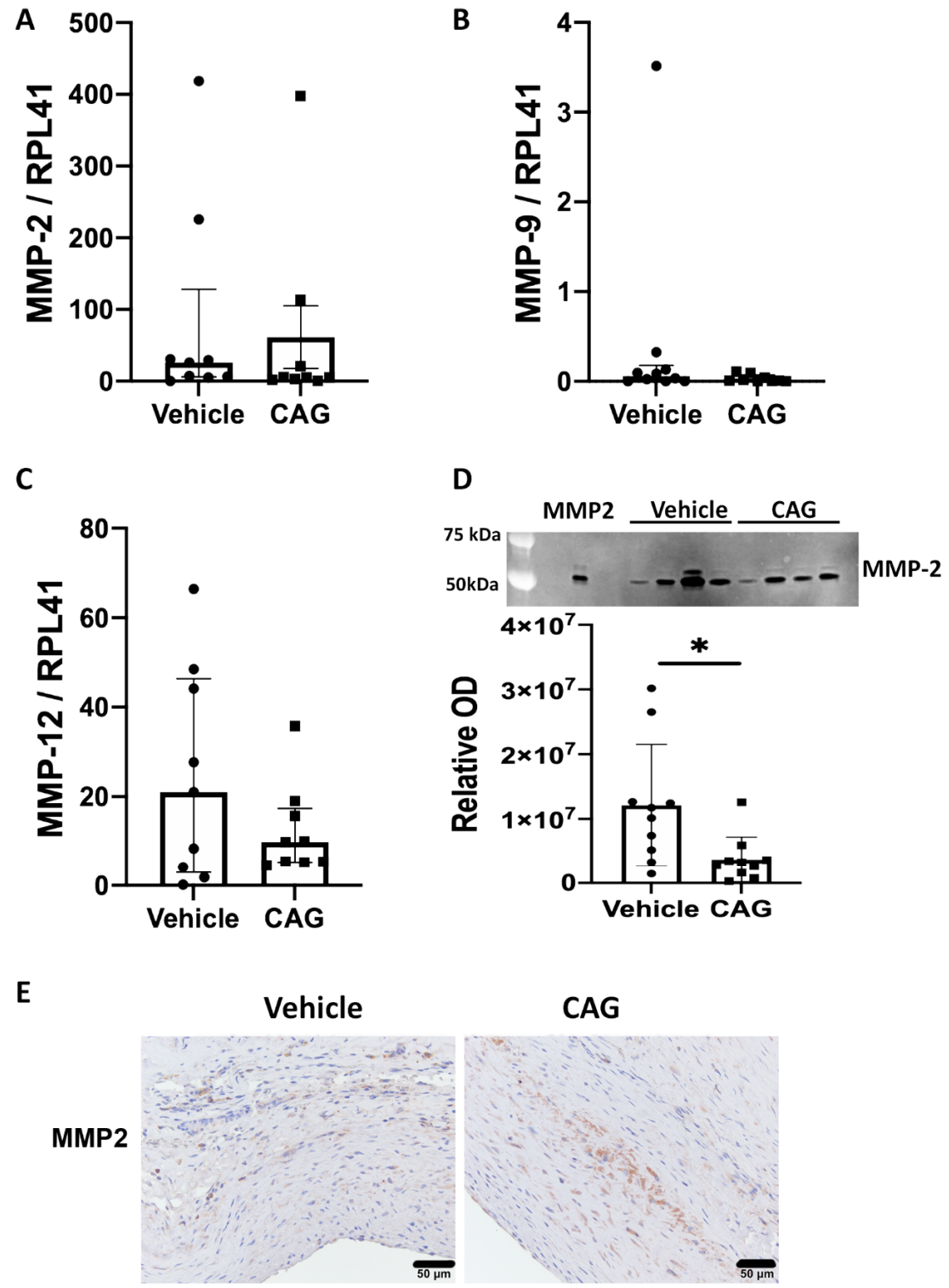

\section{CAG}

Figure 4. Quantity and activity of matrix metalloproteinases (MMPs) in AAAs. Assessment of messenger RNA (mRNA) from aneurysmal wall samples from vehicle and CAG group coding for (A) MMP-2 ( $\mathrm{n}=9 / 9)$, (B) MMP-9 ( $\mathrm{n}=9 / 9)$, and (C) MMP-12 ( $=$ = 9/9). (D) Zymography and quantification of zymography showing significantly increased activity of MMP-2 in vehicle compared to CAG group ( $\mathrm{n}=10 / 10 ; p=0.02$ ). (E) Displays weak MMP-2 labeling in a subset of vascular smooth muscle cells of the aneurysm wall from both vehicle- and CAG-treated rats $(n=8 / 7)$, scalebar $=50 \mu \mathrm{m}$. Values in (A-C) are median \pm interquartile range and normalized to RPL41 mRNA levels. Values in (D) are mean $\pm \mathrm{SD}, *$ indicates $p=0.02$. 


\subsection{The Effect of CAG on Infiltration of Inflammatory Cells into the Aneurysm Wall}

Next, we determined the suggested anti-inflammatory properties of CAG by determining infiltration of immune cells into the aneurysm wall. The mRNA levels of the common lymphocyte marker CD45 (Figure 5A, $p=0.63$ ) and the monocyte/macrophage marker F4 / 80 (Figure $5 \mathrm{~B}, p=0.44$ ) were not affected in the CAG treated aneurysms.
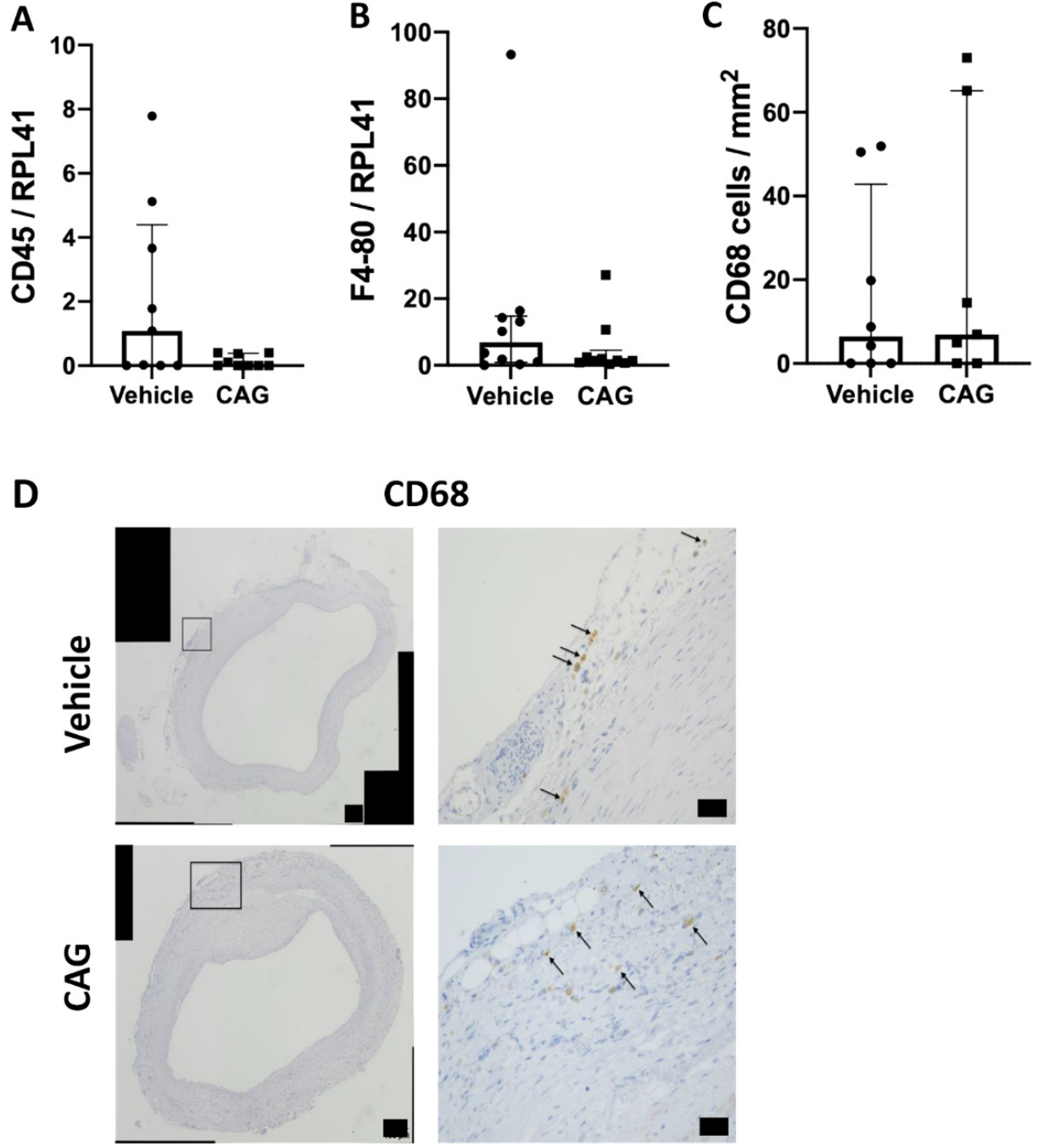

Figure 5. Immune cells in abdominal aortic aneurysms. (A) Relative mRNA levels from aneurysmal wall samples from vehicle and CAG treated group of common lymphocyte marker CD45 ( $\mathrm{n}=9 / 9)$, and (B) macrophage/monocyte marker F4-80 ( $\mathrm{n}=10 / 10)$. All RNA data were normalized to RPL41 mRNA levels. (C) Semi-quantification of aneurysmal CD68 positive cells in AAA of each group per $\mathrm{mm}^{2}$ in the adventitial layer $(\mathrm{n}=8 / 7)$. (D) Representative micrographs of CD68 positive cells in vehicle and CAG AAAs on day 28 (enlargement represents black square on the left image). Arrows mark positive cells $(n=8 / 7)$. Scale bar (black box) in micrographs to the left: $100 \mu \mathrm{m}$ and in micrographs to the left: $50 \mu \mathrm{m}$. Values are median \pm interquartile range.

The number of infiltrating macrophages identified as CD68 positive cells, localized to the adventitial layer of the aneurysms, did not show any difference between groups (Figure $5 \mathrm{C}, \mathrm{D}, p=0.56$ ).

As the balance between pro-inflammatory M1 macrophages and tissue repairing M2 macrophages has previously been shown to be important for AAA expansion [9], the M2 macrophages identified as CD206 positive cells were determined in the aneurysm wall (Figure 6A). CD206 positive cells were limited to the adventitial layer, and there were no differences in the number of CD206 positive cells per $\mathrm{mm}^{2}$ between the two groups 
(Figure 6B, $p=0.99$ ). In agreement, there were no difference in the aneurysmal mRNA levels of the anti-inflammatory cytokine IL-10 between groups (Figure $6 \mathrm{C}, p=0.114$ ). Moreover, levels of iNOS mRNA, another marker for M1 macrophages, did not differ between the vehicle and CAG treated groups (Figure 6D, $p=0.684$ ) and there was no difference in aneurysmal mRNA levels of the pro-inflammatory cytokine IL-6 between groups (Figure 6E, $p=0.340$ ). Thus, the inflammatory response seemed not to be affected by CAG treatment.

A
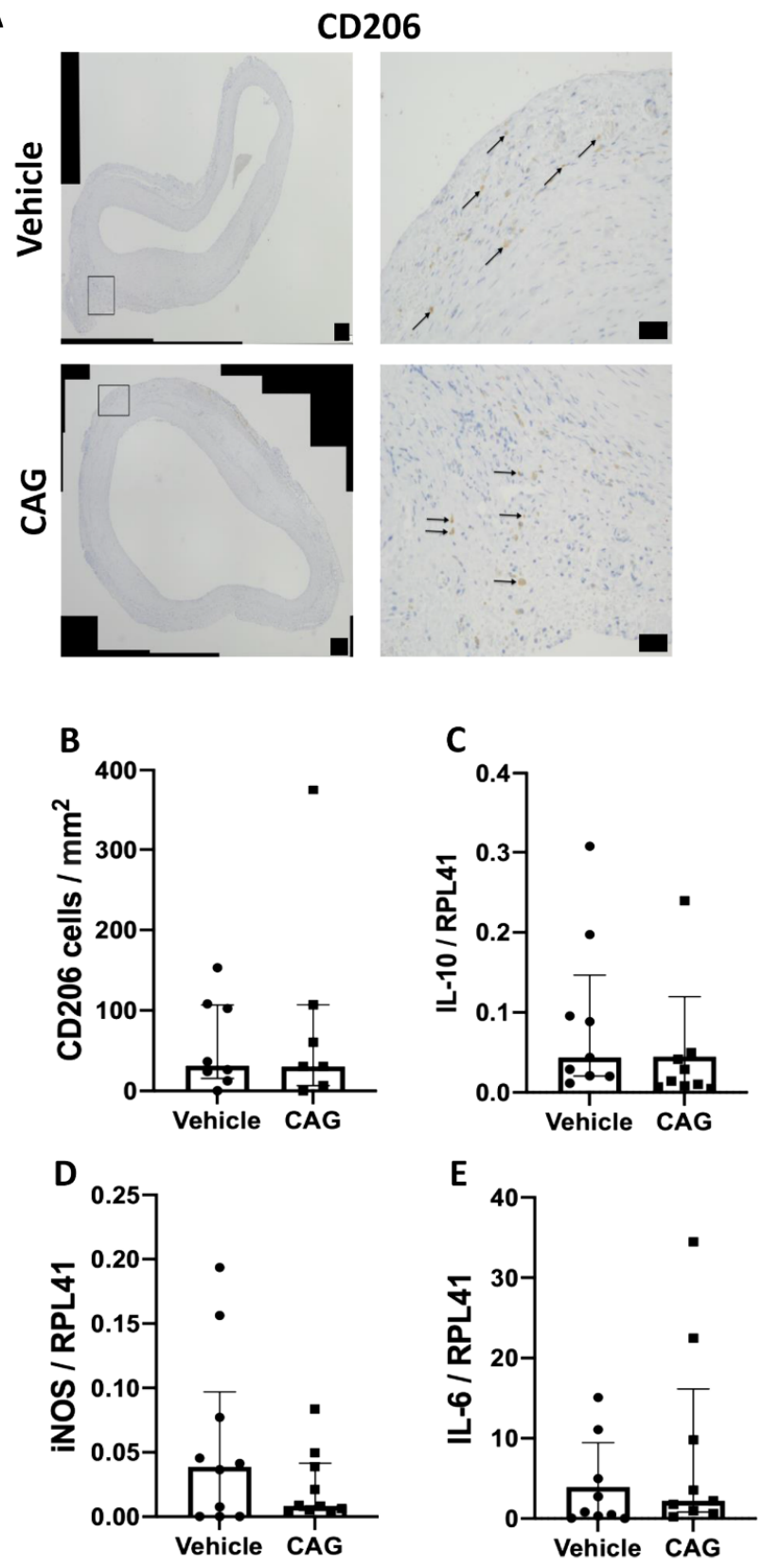

Figure 6. Markers for M1 and M2 macrophages and cytokine expression in the aneurysm wall after 28 days on vehicle or CAG treatment. (A) Representative micrographs of CD206 positive staining from vehicle and CAG AAAs at day 28 (enlargement represent black square on the left image). Arrows mark positive cells $(n=8 / 7)$. Scale bar (black box) in micrographs to the left: $100 \mu \mathrm{m}$ and in micrographs to the right: $50 \mu \mathrm{m}$. (B) Semi-quantification of CD206 positive cells in each group per $\mathrm{mm}^{2}$ in adventitia. (C) Relative mRNA levels of IL-10 $(\mathrm{n}=10 / 10),(\mathrm{D})$ inducible NO synthase (iNOS) $(\mathrm{n}=10 / 10)$, and (E) IL-6 $(\mathrm{n}=9 / 9)$ in the aneurysm tissue. All RNA data is normalized to RPL41 mRNA levels. All values are median \pm interquartile range. 


\subsection{The Effect of CAG on Oxidative Stress and Calcification of the Aneurysm Wall}

To determine if CAG dampens AAA progression by reducing oxidative stress, the levels of antioxidative marker Nrf2 mRNA and its downstream target $\mathrm{HO}-1$ were determined in the aneurysms. Neither Nrf2 nor HO-1 mRNA levels differed between treatment groups (Figure 7A,B; $p=0.171$ and $p=0.489$, respectively).
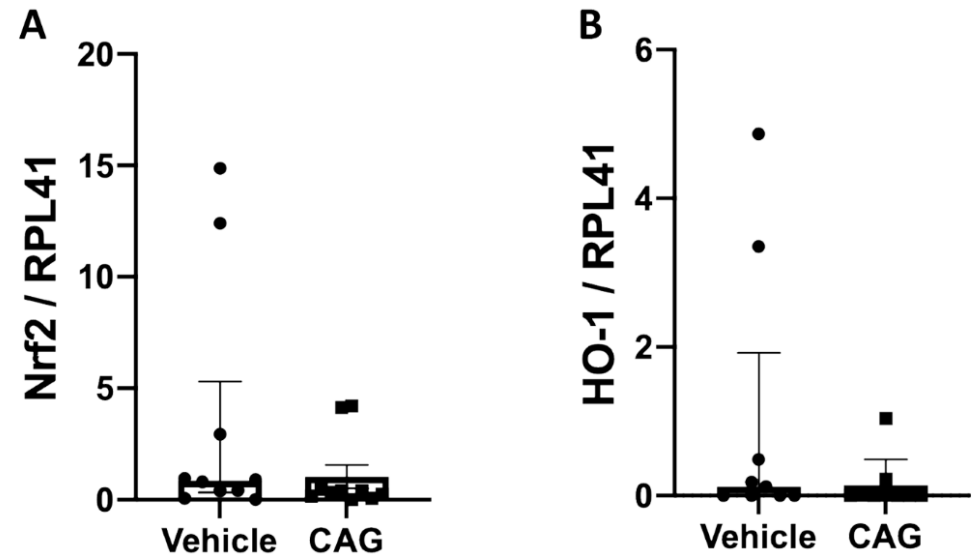

Figure 7. Effect of CAG on aneurysmal oxidative stress. (A) Relative mRNA levels of nuclear factor erythroid 2-related factor (Nrf2) $(\mathrm{n}=9 / 9)$ and (B) relative mRNA levels of Heme oxygenase (HO)-1 $(\mathrm{n}=9 / 9)$ in the aneurysm tissue. All values are median \pm interquartile range. All RNA data is normalized to RPL41 mRNA levels.

In more advanced AAAs, calcifications become significant [33]; therefore, the effect of CAG on calcifications was examined by Von Kossa's calcium deposit staining. Calcifications were present in 4 out of 7 AAA samples in the vehicle treated group, while no calcifications were detected in 7 out of 7 in the CAG-treated group (Figure $8 \mathrm{~A}, \mathrm{~B}, p=0.018$ ).

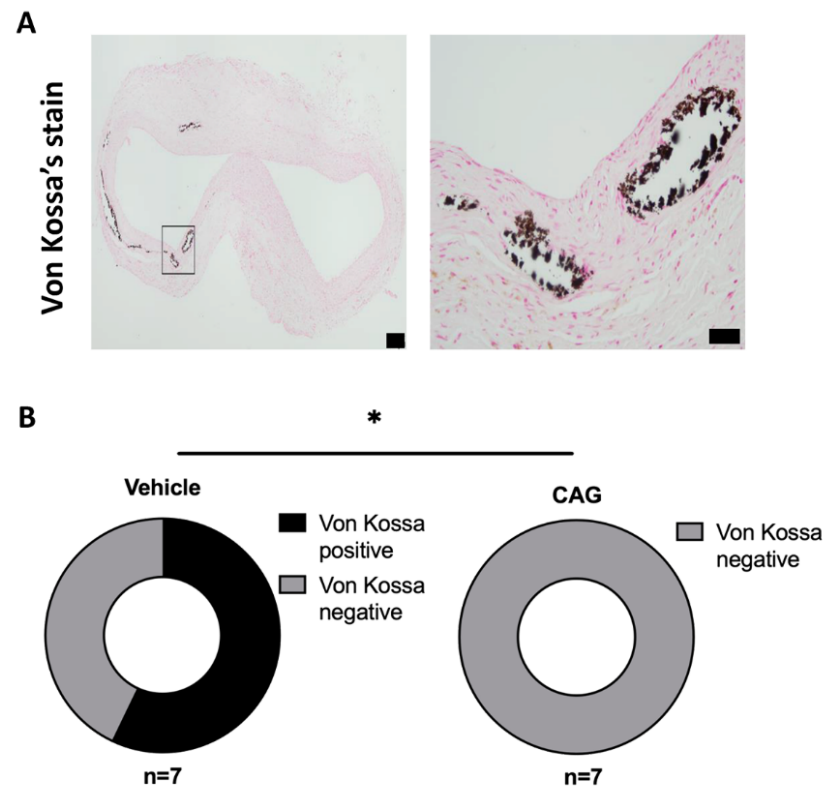

Figure 8. The effect of CAG treatment on aneurysm calcification. (A) Representative micrographs of calcium deposits (Black) in the aneurysm wall visualized by Von Kossa's staining (enlargement represent black square on the left image). Scale bar (black box) in micrograph to the left: $100 \mu \mathrm{m}$ and in micrograph to the right: $50 \mu \mathrm{m}$. ( $\mathrm{n}=7 / 7)$. (B) Donut plot of percentage of Von Kossa's positive aneurysm sections in CAG and vehicle treated groups $(n=7 / 7 ; p=0.018)$. All values are median \pm interquartile range. All RNA data is normalized to RPL41 mRNA levels. ${ }^{*}$ Indicates $p<0.05$. 


\subsection{The Effect of CAG AAA Protein Composition Using Explorative Proteomics}

To identify new mechanisms of CAG in limiting aneurysm progression, protein samples of the aneurysms were analyzed by liquid chromatography mass spectrometry (LCMS/MS). We identified 2011 unique proteins (minimum $n=3 / 3$ ), of which $57 \%$ were detected across all samples $(n=10 / 10)$ (Supplementary Table S1). No significant differences were found between CAG-treated and vehicle-treated aneurysms when correcting for multiple testing (Supplementary Figure S2); thus, one should bear in mind that some unadjusted de-regulated proteins might be false positive.

The top 20 de-regulated proteins in the aneurysm wall are shown in Table 2. The proteins identified in the aneurysm wall include the structural fibulin- 5 involved in elastin assembly [8], the anti-aggregatory and vasodilatory $\mathrm{PGI}_{2}$-producing enzyme prostacyclin synthase [34], and the water channel aquaporin-1 (AQP1). Table 2: Top 20 hits of proteins deregulated in CAG treated AAA tissue compared to vehicle treated AAA by explorative proteomics $(n=10 / 10)$. In this table, data is not adjusted for multiple testing. FC: fold change.

Table 2. Top Deregulated Proteins in CAG Treated AAA vs. Vehicle Treated AAA.

\begin{tabular}{cccc}
\hline Accession & Description & Fold Change & $p$-Value \\
\hline O08658 & Nuclear pore complex protein & 1.17 & 0.001 \\
Q9Z1X1 & Extended synaptotagmin-1 & 1.15 & 0.77 \\
P61227 & Ras-related protein Rap-2b & 0.79 & 0.003 \\
P20171 & GTPase HRas OS = Rattus norvegicus & 1.14 & 0.003 \\
P53534 & Glycogen phosphorylase, brain form (Fragment) & 1.71 & 0.004 \\
P21263 & Nestin & 1.25 & 0.004 \\
Q62969 & Prostacyclin synthase & 1.22 & 0.007 \\
O35353 & Guanine nucleotide-binding protein subunit beta-4 & 1.16 & 0.010 \\
Q4V8H8 & EH domain-containing protein 2 & 1.39 & 0.012 \\
P09414 & Nuclear factor 1 A-type & 1.19 & 0.014 \\
O89043 & DNA polymerase alpha subunit B & 0.80 & 0.014 \\
Q8CF97 & Deubiquitinating protein VCIP135 & 0.014 \\
P63029 & Translationally-controlled tumor protein & 0.90 & 0.014 \\
P29975 & Aquaporin-1 & 1.25 & 0.016 \\
Q62745 & CD81 antigen & 1.07 & 0.016 \\
B2RYW9 & Fumarylacetoacetate hydrolase domain-containing protein 2 & 1.20 & 0.018 \\
Q7TQ16 & Cytochrome b-c1 complex subunit 8 & 1.18 & 0.018 \\
P60892 & Ribose-phosphate pyrophosphokinase 1 & 1.14 & 0.019 \\
Q9JLZ1 & Glutaredoxin-3 & 0.93 & 0.020 \\
Q9WVH8 & Fibulin-5 & 1.15 & 0.020 \\
\hline
\end{tabular}

\subsection{Effect of CAG on Vascular Smooth Muscle Cells}

To determine if vascular smooth muscle cell layers in the aneurysm wall were changed by CAG treatment, the $\alpha$-actin positive cells were examined in aneurysm cross-sections from the two groups. Intense $\alpha$-actin labeling was detected in the medial layer of the aneurysms in both groups; there was no difference in the area of positive $\alpha$-actin staining between vehicle and CAG-treated rats (Figure $9 \mathrm{~A}, \mathrm{~B}, p=0.56$ ). That no major change in vascular smooth muscle cell layer was observed was further supported by the quantitative proteome analyses of the aneurysm wall showing no change in proteins associated with vascular smooth muscle cells contractile phenotype [35]; myosin 11, $\alpha$-actin, transgelin/SM22, calponin-1, myosin regulatory light polypeptide 9 , and topomyosin $\beta$ chain (Supplementary Table S2 and Supplementary Figure S2, yellow dots) between vehicle and CAG-treated rats. 
A

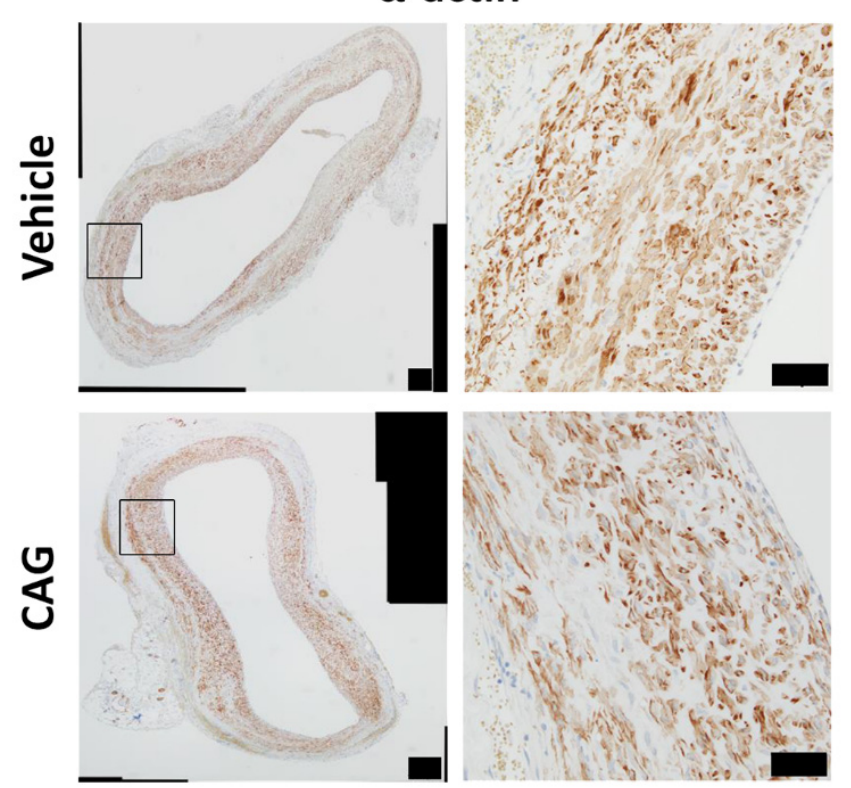

B

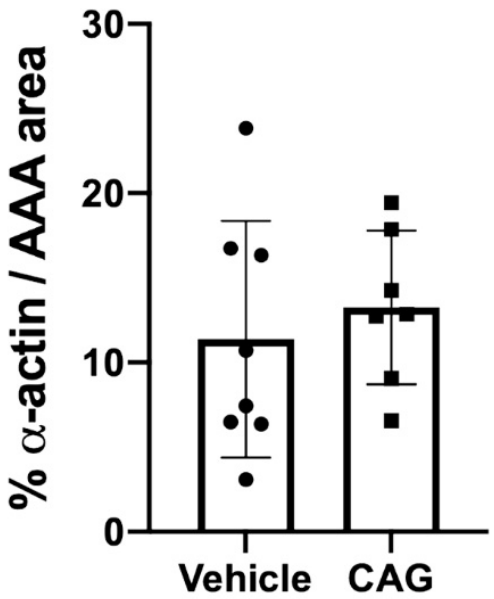

Figure 9. CAG does not affect $\alpha$-actin positive area in the aneurysm wall. (A) Representative micrographs of $\alpha$-actin staining from vehicle and CAG AAAs at day 28 (enlargement represent black square on the left image, $(n=8 / 7)$. Scale bar (black box) in micrographs to the left: $100 \mu \mathrm{m}$ and in micrographs to the right: $50 \mu \mathrm{m}$. (B) Semi-quantification of $\alpha$-actin positive area of total AAA area. Values are mean \pm SD.

\section{Discussion}

In the present study, we aimed to test the proposed protective effects of CAG supplementation on AAA progression. We found that daily administration of CAG significantly attenuated expansion of intraluminal elastase-induced AAA in rats. The aneurysms displayed more preserved elastic lamellae. The underlying mechanism could be linked to diminished aneurysmal MMP-2 activity.

Preservation of elastic lamellae in the CAG treated AAAs could be explained by decreased degradation of elastin or augmented synthesis. Our data suggest that it may not be caused by increased elastin synthesis, as LOX mRNA levels were unchanged. However, we did detect a non-significant upregulation of fibulin- 5 by our explorative proteome analysis (FC 1.15 and unadjusted $p$-value $=0.020$ ). Both LOX and fibulin-5 enable the formation of elastin fibers in the aorta by binding to structural proteins such as tropoelastin and fibrillin-1, thereby facilitating increased elastin assembly in AAAs [8]. Moreover, in cultured rat vascular smooth muscle cells, CAG restored the TNF-mediated reduction in expression of fibulin-5 and -1 [19]. The effect of CAG is, however, more likely caused by decreased MMP-2 activity in the CAG-treated aneurysms produced in vascular smooth muscle cells. This is in line with previous murine studies showing a reduced AAA expansion associated with decreased MMP activity, both in the murine elastase wrapping model and the angiotensin II-induced AAA model in hyperlipidemic $\mathrm{ApoE}^{-/-}$mice [19]. That CAG directly affects vascular smooth muscle cells and, thereby inhibits MMP activity, has been shown in TNF-stimulated cultured primary rat vascular smooth muscle cells; the affected molecular signaling pathway was ascribed to dampening of the ERK/JNK signaling pathway [19].

One of the critical elements in AAA progression includes chronic inflammation associated with continuous infiltration of macrophages and lymphocytes because of degradation of the ECM in the aneurysm wall. The infiltrated immune cells release pro-inflammatory cytokines and proteases that activate VSMCs to phenotypic shift and increased collagen production as a compensatory mechanism for the degradation of elastin fibers. Eventually VSMCs become stressed and undergo apoptosis resulting in high production of RNS and 
ROS [36-39]. In murine models, inflammation is the primary driver of AAA progression in the first two weeks of AAA expansion [40,41]; thus, this could explain why we, in this study, did not observe any effect of CAG on the monocyte/macrophage marker F4/80 at the mRNA level, along with no reduction of CD68 positive macrophages in the wall of the CAG treated aneurysms. A reduction in infiltrating CD68 positive cells in the aneurysm wall has previously been observed after CAG treatment in aneurysms in mice induced by local elastase wrapping around the abdominal aorta, and in mice where the component 3,4-benzopyrene (an active ingredient in cigarettes) enhanced angiotensin II AAA two weeks, but also six weeks, after AAA induction $[19,42]$. The underlying mechanisms were ascribed to reduction in transforming growth factor beta (TGF $\beta$ ) and nuclear factor kBinduced production of pro-inflammatory cytokines resulting in diminished inflammation. A similar effect of CAG on macrophages was observed in chronic psoriatic skin lesions in mice, where CAG administration reduced infiltration of macrophages and decreased mRNA levels of the pro-inflammatory cytokines IL-1 $\beta, T N F$, and IL-6 in the inflamed skin and in LPS stimulated bone marrow-derived macrophages in vitro. The effect of CAG was shown to dampen inflammation by preventing NRLP3 inflammasome activation in bone marrow derived macrophages [18].

As there is consensus that CAG seems to inhibit macrophage infiltration and expression of pro-inflammatory cytokines, we would have expected to detect lower mRNA expression levels of IL-6 in the CAG-treated aneurysms. IL-6 is believed to act as a chemoattractant for immune cells in aneurysms. However, it has not been proven to individually affect progression or expansion of AAAs in mice [43]. Our findings of no difference in IL-6 expression between groups contradicts the existing literature [19]. IL-6 belongs to one of the early inflammatory response genes in inflamed tissue [44]. However, we examined the aneurysmal tissue after 28 days, a phase that, in rats, is more regenerative [40]; macrophages shift to favor the anti-inflammatory and tissue repairing phenotype [12,45], which could be why we did not detect any difference in IL-6 levels.

The balance between M1 and M2 macrophages is important for tissue homeostasis [12]. Dale et al. demonstrated that favoring M1 macrophages augmented AAA expansion, while favoring M2 macrophages dampened AAA progression [9]. Thus, we expected that CAG administration would favor the M2 phenotype. We did not find any difference between groups in our semi-quantifications of CD206 positive cells in aneurysm wall cross-sections. In addition, the mRNA levels of the anti-inflammatory cytokine IL-10 in the aneurysms were not significantly different, nor were mRNA levels of iNOS used as an indicator of M1 macrophages in the aneurysms. Thus, in our study, CAG did not seem to favor a shift from M1 to M2 macrophages. No change in inflammatory status was observed in the aneurysm wall. This corresponds with the absence of changes in the media layer of $\alpha$-actin positive cells and the fact that we did not detect any difference in quantitative proteins associated with vascular smooth muscle cell contractile phenotype.

As previously described, ROS contribute significantly to AAA progression in both human and murine models $[46,47]$ as ROS promote macrophages to release pro-inflammatory cytokines such as IL-6. Astragaloside IV (AST) obtained from Astragalus membranaceus is easily converted by intestinal microbes to CAG by deglycosylation [48]. In murine models of AAA, both AST and CAG reduced ROS and, thereby, dampened the release of proinflammatory cytokines IL-6, TNF and MMPs from macrophages [19,42]. The underlying mechanism relates to augmented Nrf2 and HO-1 signaling pathways. Nrf2 is a transcription factor that controls the expression of antioxidant genes [10]. Thus, upregulation of Nrf2 will diminish ROS and, thereby, ROS-mediated inflammation [46]. HMOX1 is a cellular stress response gene regulated by $\mathrm{Nrf} 2$ that produces $\mathrm{HO}-1$. $\mathrm{HO}-1$ is responsible for the oxidative cleavage of heme groups released from damaged erythrocytes in the vascular wall, leading to the generation of biliverdin with antioxidant properties, thereby scavenging ROS, carbon monoxide with vasodilatory properties, and release of ferrous iron. Thus, HO-1 has important antioxidant, anti-inflammatory, and cytoprotective effects in vascular cells. Thus, the presence of HO-1 protects vascular smooth muscle cells and endothelial 
cells from further damage in response to injury. Furthermore, HO-1 deficiency in mice augments AAA progression [49]. Moreover, in humans, polymorphisms in the promoter region of the HMOX1 gene, resulting in decreased expression of $\mathrm{HO}-1$, are associated with increased risk of developing AAA [50]. In our experiments, neither Nrf2 nor HO-1 mRNA levels changed after CAG treatment, suggesting that, in our setting, CAG did not influence ROS or exert antioxidative effects at the examined time point. Perhaps, this could be explained by species difference or the dose of CAG used. The studies showing that CAG or AST reduced the expression of $\mathrm{Nrf} 2$ and $\mathrm{HO}-1$ were performed in mice using two different models: the elastase wrapping model and the angiotensin II and 3,4 benzopyrene-induced AAA model in 8-10 months old C57BL/6 mice. As in our experiments, CAG was given orally. In the angiotensin II and 3.4-benzopyrene model, they used daily doses of $20 \mathrm{mg} / \mathrm{kg}$ and $80 \mathrm{mg} / \mathrm{kg}$ for 6 weeks [42], while CAG, in the elastase wrapping model, was given in a low dose $62.5 \mathrm{mg} / \mathrm{kg}$ or high dose $125 \mathrm{mg} / \mathrm{kg}$ daily for 14 or 28 days perorally, starting at the day of experiment or at 14 days (high dose only) after AAA induction [19]. We initiated our CAG administration the day after surgery to prevent pre-priming of the aortic wall prior to elastase treatment. The high dose in our experiment was chosen based on the daily dose of CAG on an oral no-observed-adverse-effect-level (NOAEL) > $150 \mathrm{mg} /$ day in rats, achieved by oral administration of $150 \mathrm{mg}$ CAG/ kg/day for 91 consecutive days [51] and corresponded to the dose used in Wang et al. [19], though there might be species difference.

To find new potential mechanisms that CAG might affect to dampen AAA progression, we used an explorative proteome approach. We did not find any deregulated proteins in the aneurysm wall in comparing the two groups, when adjustment for multiple testing was done. This might reflect the highly heterogenous tissue that requires numerous samples to detect differences. Amongst the unadjusted regulated genes with a $p$-value below 0.02 was prostacyclin synthase (PGIS) (FC 1.27, unadjusted $p$-value $=0.006$ ). PGIS produces prostacyclin with known vasodilatory, anti-inflammatory, and anti-thrombotic properties counteracting the prothrombotic thromboxane $[34,52,53]$. The water channel AQP1 was also upregulated (FC 1.26, unadjusted $p$-value $=0.01$ ). We have previously shown that loss of AQP1 accelerates angiotensin II-induced atherosclerosis in hyperlipidemic mice [29]. The underlying mechanism was not identified; however, AQP1 channels in the endothelial cells could, perhaps, contribute to washing out substances, such as LDL, trapped in the subendothelial intimal layer in areas of endothelial dysfunction.

The protective effect of CAG could also be mediated by lowering arterial blood pressure. We did not measure blood pressure in our study, but we did observe a difference in heart to body weight ratios, with a higher ratio among CAG-treated rats that could suggest an elevated blood pressure. However, we believe that the elevated heart to body ratio more likely relates to the relatively lower body weights within the CAG-treated rats, as liver and spleen to body ratios were also slightly elevated. Others have reported that the compound astragaloside IV, which is converted by intestinal microbes to CAG [54], did not affect arterial blood pressure in pregnant rats [55]. They did, however, observe a dosedependent (20-80 mg/kg) blood pressure lowering effect in preeclampsia-induced pregnant rats, as well as a reduction in preeclampsia-induced oxidative stress [55], suggesting that CAG in our model could potentially have a minor blood pressure lowering effect rather than elevating blood pressure. In comparison, we treated our rats with a higher dose of CAG that seemed to be well tolerated; the rats had a similar weight gain as the vehicle treated controls, in line with the existing literature [19,51].

There are some limitations to the study. Our primary end point was progressive aneurysm dilatation. Therefore, we chose the PPE AAA model in rats, as it is, to our knowledge, the model that presents most of the features of the human disease $[20,40,41]$. All models are short term, while human AAA develops over years [1,6]. The length of the experimental protocol is, in most studies, either 14 days or 28 days. The first 14 days of AAA expansion is fastest and involves inflammation and oxidative stress as drivers [56]. While, in the last 14 days, AAA expansion declines and reaches a plateau, reflecting tissuerepairing mechanisms with extensive elastin production. To get the full effect of CAG on 
AAA expansion, we chose to end the experiment after 28 days, when AAA size and elastin integrity could be analyzed, while proinflammatory responses may be less pronounced. Although the reduction in MMP-2 activity seen at day 28 in the aneurysm wall may reflect reduced inflammation and/or oxidative stress at an earlier timepoint.

The effect of CAG in already established AAA has yet to be evaluated. This would be clinically relevant. Although, in our experiments, CAG treatment was provided after induction of AAA by elastase infusion. Thus, we did not affect the aortic wall by initiating CAG treatment prior to AAA induction, which suggests that CAG could likely be beneficial in existing AAA and is supported by findings in mice [19].

\section{Conclusions}

In conclusion, CAG reduced experimental AAA progression. Our data suggest that underlying mechanisms might be mediated by reduced MMP- 2 activity and by preserving elastin and reduced calcification. Based on these findings, CAG should be considered as a possible candidate for future dietary supplementation that may dampen AAA expansion in humans.

Supplementary Materials: The following supporting information can be downloaded at: https:// www.mdpi.com/article/10.3390/biomedicines10020359/s1, Figure S1: Body weight changes and tissue weights after 28 days of CAG treatment. Figure S2: Volcano plot showing changes in aneurysmal protein levels between CAG treated and vehicle treated groups using proteomic analyses. Table S1:All identified proteins in CAG vs. vehicle treated AAAs. Table S2: CAG treatment does not affect aneurysm protein levels related to VSMC contractile phenotype compared to vehicle treated rats.

Author Contributions: Conceptualization and methodology: L.G.M., J.H.D., J.S.L. and J.S.; software, validation, formal analysis, resources, data curation and visualization: L.G.M., J.H.D., L.B.S., S.L.E., P.D.C., H.C.B., J.S.L. and J.S.; writing-original draft preparation: L.G.M., L.B.S., J.S.L. and J.S.; writing - review and editing: L.G.M., L.B.S., J.H.D., J.S.L., H.C.B., S.L.E., P.D.C., L.M.R. and J.S.; supervision and project administration: J.S., J.S.L. and L.G.M.; Funding acquisition: J.S.L., L.G.M. and J.S. All authors have read and agreed to the published version of the manuscript.

Funding: The research was funded by The Region of Southern Denmark (19/25225), A. P. Moeller Foundation (19-L-0069) and Novo Nordisk Foundation (NNF19OC0058959).

Institutional Review Board Statement: Not applicable. All animal experiments were as described in the method section conducted as according to an animal protocol approved by the Danish Animal Experiments Inspectorate (license nr. 2016-15-0201-01046).

Informed Consent Statement: Not applicable.

Data Availability Statement: The mass spectrometry proteomics data have been deposited to the ProteomeXchange Consortium via the PRIDE [57] partner repository with the dataset identifier PXD030441.

Acknowledgments: We would like to thank our skillful technicians Lene Bundgaard Andersen, Amalie Kamstrup Mogensen, Kenneth Kjærsgaard, Inger Nissen, Bianca Jørgensen, Anne Mette Durand, and Maja Friis Waltersdorff for their excellent technical assistance. We thank Anthony M. Carter for linguistic correction.

Conflicts of Interest: The authors have no conflict of interest. The funders had no role in the design of the study; in the collection, analyses, or interpretation of data; in the writing of the manuscript; or in the decision to publish the results.

\section{References}

1. Kuivaniemi, H.; Ryer, E.J.; Elmore, J.R.; Tromp, G. Understanding the pathogenesis of abdominal aortic aneurysms. Expert Rev. Cardiovasc. Ther. 2015, 13, 975-987. [CrossRef] [PubMed]

2. Howard, D.P.; Banerjee, A.; Fairhead, J.F.; Handa, A.; Silver, L.E.; Rothwell, P.M. Population-Based Study of Incidence of Acute Abdominal Aortic Aneurysms With Projected Impact of Screening Strategy. J. Am. Heart Assoc. 2015, 4, e001926. [CrossRef] [PubMed]

3. Filardo, G.; Powell, J.T.; Martinez, M.A.; Ballard, D.J. Surgery for small asymptomatic abdominal aortic aneurysms. Cochrane Database Syst Rev. 2015, 2015, Cd001835. [CrossRef] [PubMed] 
4. Kent, K.C. Clinical practice. Abdominal aortic aneurysms. N. Engl. J. Med. 2014, 371, 2101-2108. [CrossRef] [PubMed]

5. Moll, F.L.; Powell, J.T.; Fraedrich, G.; Verzini, F.; Haulon, S.; Waltham, M.; van Herwaarden, J.A.; Holt, P.J.; van Keulen, J.W.; Rantner, B.; et al. Management of abdominal aortic aneurysms clinical practice guidelines of the European society for vascular surgery. Eur. J. Vasc. Endovasc. Surg. 2011, 41 (Suppl. 1), S1-S58. [CrossRef] [PubMed]

6. Ailawadi, G.; Eliason, J.L.; Upchurch, G.R., Jr. Current concepts in the pathogenesis of abdominal aortic aneurysm. J. Vasc. Surg. 2003, 38, 584-588. [CrossRef]

7. Campa, J.S.; Greenhalgh, R.M.; Powell, J.T. Elastin degradation in abdominal aortic aneurysms. Atherosclerosis 1987, 65, 13-21. [CrossRef]

8. Yanagisawa, H.; Schluterman, M.K.; Brekken, R.A. Fibulin-5, an integrin-binding matricellular protein: Its function in development and disease. J. Cell Commun. Signal. 2009, 3, 337-347. [CrossRef]

9. Dale, M.A.; Xiong, W.; Carson, J.S.; Suh, M.K.; Karpisek, A.D.; Meisinger, T.M.; Casale, G.P.; Baxter, B.T. Elastin-Derived Peptides Promote Abdominal Aortic Aneurysm Formation by Modulating M1/M2 Macrophage Polarization. J. Immunol. 2016, 196, $4536-4543$. [CrossRef]

10. Kovac, S.; Angelova, P.R.; Holmström, K.M.; Zhang, Y.; Dinkova-Kostova, A.T.; Abramov, A.Y. Nrf2 regulates ROS production by mitochondria and NADPH oxidase. Biochim. Biophys. Acta 2015, 1850, 794-801. [CrossRef]

11. Chen, Q.; Wang, Q.; Zhu, J.; Xiao, Q.; Zhang, L. Reactive oxygen species: Key regulators in vascular health and diseases. Br. J. Pharmacol. 2018, 175, 1279-1292. [CrossRef] [PubMed]

12. Dale, M.A.; Ruhlman, M.K.; Baxter, B.T. Inflammatory cell phenotypes in AAAs: Their role and potential as targets for therapy. Arterioscler. Thromb. Vasc. Biol. 2015, 35, 1746-1755. [CrossRef] [PubMed]

13. McCormick, M.L.; Gavrila, D.; Weintraub, N.L. Role of oxidative stress in the pathogenesis of abdominal aortic aneurysms Arterioscler. Thromb. Vasc. Biol. 2007, 27, 461-469. [CrossRef] [PubMed]

14. Yu, Y.; Zhou, L.; Yang, Y.; Liu, Y. Cycloastragenol: An exciting novel candidate for age-associated diseases. Exp. Ther. Med. 2018, 16, 2175-2182. [CrossRef]

15. Sun, C.; Jiang, M.; Zhang, L.; Yang, J.; Zhang, G.; Du, B.; Ren, Y.; Li, X.; Yao, J. Cycloastragenol mediates activation and proliferation suppression in concanavalin A-induced mouse lymphocyte pan-activation model. Immunopharmacol. Immunotoxicol. 2017, 39, 131-139. [CrossRef]

16. Wang, J.; Wu, M.L.; Cao, S.P.; Cai, H.; Zhao, Z.M.; Song, Y.H. Cycloastragenol ameliorates experimental heart damage in rats by promoting myocardial autophagy via inhibition of AKT1-RPS6KB1 signaling. Biomed. Pharmacother. 2018, 107, 1074-1081. [CrossRef]

17. Gu, M.; Zhang, S.; Zhao, Y.; Huang, J.; Wang, Y.; Li, Y.; Fan, S.; Yang, L.; Ji, G.; Tong, Q.; et al. Cycloastragenol improves hepatic steatosis by activating farnesoid $X$ receptor signalling. Pharmacol. Res. 2017, 121, 22-32. [CrossRef]

18. Deng, G.; Chen, W.; Wang, P.; Zhan, T.; Zheng, W.; Gu, Z.; Wang, X.; Ji, X.; Sun, Y. Inhibition of NLRP3 inflammasome-mediated pyroptosis in macrophage by cycloastragenol contributes to amelioration of imiquimod-induced psoriasis-like skin inflammation in mice. Int. Immunopharmacol. 2019, 74, 105682. [CrossRef]

19. Wang, Y.; Chen, C.; Wang, Q.; Cao, Y.; Xu, L.; Qi, R. Inhibitory effects of cycloastragenol on abdominal aortic aneurysm and its related mechanisms. Br. J. Pharmacol. 2019, 176, 282-296. [CrossRef]

20. Anidjar, S.; Salzmann, J.L.; Gentric, D.; Lagneau, P.; Camilleri, J.P.; Michel, J.B. Elastase-induced experimental aneurysms in rats. Circulation 1990, 82, 973-981. [CrossRef]

21. Lysgaard Poulsen, J.; Stubbe, J.; Lindholt, J.S. Animal Models Used to Explore Abdominal Aortic Aneurysms: A Systematic Review. Eur. J. Vasc. Endovasc. Surg. 2016, 52, 487-499. [CrossRef] [PubMed]

22. Kilkenny, C.; Browne, W.; Cuthill, I.C.; Emerson, M.; Altman, D.G.; Group NCRRGW. Animal research: Reporting in vivo experiments: The ARRIVE guidelines. Br. J. Pharmacol. 2010, 160, 1577-1579. [CrossRef] [PubMed]

23. Johnston, W.F.; Salmon, M.; Su, G.; Lu, G.; Ailawadi, G.; Upchurch, G.R., Jr. Aromatase is required for female abdominal aortic aneurysm protection. J. Vasc. Surg. 2015, 61, 1565-1574.e4. [CrossRef] [PubMed]

24. Ailawadi, G.; Eliason, J.L.; Roelofs, K.J.; Sinha, I.; Hannawa, K.K.; Kaldjian, E.P.; Lu, G.; Henke, P.K.; Stanley, J.C.; Weiss, S.J.; et al. Gender differences in experimental aortic aneurysm formation. Arterioscler. Thromb. Vasc. Biol. 2004, 24, 2116-2122. [CrossRef]

25. Abelson, K.S.; Jacobsen, K.R.; Sundbom, R.; Kalliokoski, O.; Hau, J. Voluntary ingestion of nut paste for administration of buprenorphine in rats and mice. Lab. Anim. 2012, 46, 349-351. [CrossRef]

26. Schack, A.S.; Stubbe, J.; Steffensen, L.B.; Mahmoud, H.; Laursen, M.S.; Lindholt, J.S. Intraluminal infusion of Penta-Galloyl Glucose reduces abdominal aortic aneurysm development in the elastase rat model. PLoS ONE 2020, 15, e0234409. [CrossRef]

27. Steffensen, L.B.; Stubbe, J.; Lindholt, J.S.; Beck, H.C.; Overgaard, M.; Bloksgaard, M.; Genovese, F.; Nielsen, S.H.; Tha, M.L.T.; Bang-Moeller, S.K.; et al. Basement membrane collagen IV deficiency promotes abdominal aortic aneurysm formation. Sci. Rep. 2021, 11, 12903. [CrossRef]

28. Mulorz, J.; Spin, J.M.; Beck, H.C.; Tha Thi, M.L.; Wagenhäuser, M.U.; Rasmussen, L.M.; Lindholt, J.S.; Tsao, P.S.C.; Steffensen, L.B. Hyperlipidemia does not affect development of elastase-induced abdominal aortic aneurysm in mice. Atherosclerosis 2020, 311, 73-83. [CrossRef]

29. Wintmo, P.; Johansen, S.H.; Hansen, P.B.L.; Lindholt, J.S.; Urbonavicius, S.; Rasmussen, L.M.; Bie, P.; Jensen, B.L.; Stubbe, J. The water channel AQP1 is expressed in human atherosclerotic vascular lesions and AQP1 deficiency augments angiotensin II-induced atherosclerosis in mice. Acta Physiol. 2017, 220, 446-460. [CrossRef] 
30. Huang da, W.; Sherman, B.T.; Lempicki, R.A. Systematic and integrative analysis of large gene lists using DAVID bioinformatics resources. Nat. Protoc. 2009, 4, 44-57. [CrossRef]

31. Huang da, W.; Sherman, B.T.; Lempicki, R.A. Bioinformatics enrichment tools: Paths toward the comprehensive functional analysis of large gene lists. Nucleic Acids Res. 2009, 37, 1-13. [CrossRef] [PubMed]

32. Rabkin, S.W. The Role Matrix Metalloproteinases in the Production of Aortic Aneurysm. Prog. Mol. Biol. Transl. Sci. 2017, 147, $239-265$. [PubMed]

33. Buijs, R.V.; Willems, T.P.; Tio, R.A.; Boersma, H.H.; Tielliu, I.F.; Slart, R.H.; Zeebregts, C.J. Calcification as a risk factor for rupture of abdominal aortic aneurysm. Eur. J. Vasc. Endovasc. Surg. 2013, 46, 542-548. [CrossRef] [PubMed]

34. Moncada, S.; Vane, J.R. The role of prostacyclin in vascular tissue. Fed. Proc. 1979, 38, 66-71.

35. Wirka, R.C.; Wagh, D.; Paik, D.T.; Pjanic, M.; Nguyen, T.; Miller, C.L.; Kundu, R.; Nagao, M.; Coller, J.; Koyano, T.K.; et al. Atheroprotective roles of smooth muscle cell phenotypic modulation and the TCF21 disease gene as revealed by singlecell analysis. Nat. Med. 2019, 25, 1280-1289. [CrossRef]

36. Li, P.F.; Dietz, R.; von Harsdorf, R. Reactive oxygen species induce apoptosis of vascular smooth muscle cell. FEBS Lett. 1997, 404, 249-252. [CrossRef]

37. Morimoto, K.; Hasegawa, T.; Tanaka, A.; Wulan, B.; Yu, J.; Morimoto, N.; Okita, Y.; Okada, K. Free-radical scavenger edaravone inhibits both formation and development of abdominal aortic aneurysm in rats. J. Vasc. Surg. 2012, 55, 1749-1758. [CrossRef]

38. Peppin, G.J.; Weiss, S.J. Activation of the endogenous metalloproteinase, gelatinase, by triggered human neutrophils. Proc. Natl. Acad. Sci. USA 1986, 83, 4322-4326. [CrossRef]

39. Rajagopalan, S.; Meng, X.P.; Ramasamy, S.; Harrison, D.G.; Galis, Z.S. Reactive oxygen species produced by macrophage-derived foam cells regulate the activity of vascular matrix metalloproteinases in vitro. Implications for atherosclerotic plaque stability. $J$. Clin. Investig. 1996, 98, 2572-2579. [CrossRef]

40. Sénémaud, J.; Caligiuri, G.; Etienne, H.; Delbosc, S.; Michel, J.B.; Coscas, R. Translational Relevance and Recent Advances of Animal Models of Abdominal Aortic Aneurysm. Arterioscler. Thromb. Vasc. Biol. 2017, 37, 401-410. [CrossRef]

41. Pyo, R.; Lee, J.K.; Shipley, J.M.; Curci, J.A.; Mao, D.; Ziporin, S.J.; Ennis, T.L.; Shapiro, S.D.; Senior, R.M.; Thompson, R.W Targeted gene disruption of matrix metalloproteinase-9 (gelatinase B) suppresses development of experimental abdominal aortic aneurysms. J. Clin. Investig. 2000, 105, 1641-1649. [CrossRef] [PubMed]

42. Wang, J.; Zhou, Y.; Wu, S.; Huang, K.; Thapa, S.; Tao, L.; Wang, J.; Shen, Y.; Wang, J.; Xue, Y.; et al. Astragaloside IV Attenuated 3,4-Benzopyrene-Induced Abdominal Aortic Aneurysm by Ameliorating Macrophage-Mediated Inflammation. Front. Pharmacol. 2018, 9, 496. [CrossRef] [PubMed]

43. Nishihara, M.; Aoki, H.; Ohno, S.; Furusho, A.; Hirakata, S.; Nishida, N.; Ito, S.; Hayashi, M.; Imaizumi, T.; Fukumoto, Y. The role of IL-6 in pathogenesis of abdominal aortic aneurysm in mice. PLoS ONE 2017, 12, e0185923. [CrossRef] [PubMed]

44. Tanaka, T.; Narazaki, M.; Kishimoto, T. IL-6 in inflammation, immunity, and disease. Cold Spring Harb. Perspect. Biol. 2014, 6, a016295. [CrossRef] [PubMed]

45. Cheng, Z.; Zhou, Y.Z.; Wu, Y.; Wu, Q.Y.; Liao, X.B.; Fu, X.M.; Zhou, X.M. Diverse roles of macrophage polarization in aortic aneurysm: Destruction and repair. J. Transl. Med. 2018, 16, 354. [CrossRef] [PubMed]

46. Emeto, T.I.; Moxon, J.V.; Au, M.; Golledge, J. Oxidative stress and abdominal aortic aneurysm: Potential treatment targets. Clin. Sci. 2016, 130, 301-315. [CrossRef] [PubMed]

47. Usui, F.; Shirasuna, K.; Kimura, H.; Tatsumi, K.; Kawashima, A.; Karasawa, T.; Yoshimura, K.; Aoki, H.; Tsutsui, H.; Noda, T.; et al. Inflammasome activation by mitochondrial oxidative stress in macrophages leads to the development of angiotensin II-induced aortic aneurysm. Arterioscler. Thromb. Vasc. Biol. 2015, 35, 127-136. [CrossRef]

48. Ran, R.; Zhang, C.; Li, R.; Chen, B.; Zhang, W.; Zhao, Z.; Fu, Z.; Du, Z.; Du, X.; Yang, X.; et al. Evaluation and Comparison of the Inhibition Effect of Astragaloside IV and Aglycone Cycloastragenol on Various UDP-Glucuronosyltransferase (UGT) Isoforms. Molecules 2016, 21, 1616. [CrossRef]

49. Azuma, J.; Wong, R.J.; Morisawa, T.; Hsu, M.; Maegdefessel, L.; Zhao, H.; Kalish, F.; Kayama, Y.; Wallenstein, M.B.; Deng, A.C.; et al Heme Oxygenase-1 Expression Affects Murine Abdominal Aortic Aneurysm Progression. PLoS ONE 2016, 11, e0149288. [CrossRef]

50. Schillinger, M.; Exner, M.; Mlekusch, W.; Domanovits, H.; Huber, K.; Mannhalter, C.; Wagner, O.; Minar, E. Heme oxygenase-1 gene promoter polymorphism is associated with abdominal aortic aneurysm. Thromb. Res. 2002, 106, 131-136. [CrossRef]

51. Szabo, N.J. Dietary safety of cycloastragenol from Astragalus spp.: Subchronic toxicity and genotoxicity studies. Food Chem. Toxicol. 2014, 64, 322-334. [CrossRef] [PubMed]

52. Canaud, B.; Mion, C.; Arujo, A.; N'Guyen, Q.V.; Paleyrac, G.; Hemmendinger, S.; Cazenave, J.P. Prostacyclin (epoprostenol) as the sole antithrombotic agent in postdilutional hemofiltration. Nephron 1988, 48, 206-212. [CrossRef] [PubMed]

53. Stitham, J.; Midgett, C.; Martin, K.A.; Hwa, J. Prostacyclin: An inflammatory paradox. Front. Pharmacol. 2011, 2, 24. [CrossRef] [PubMed]

54. Zhao, Y.; Li, Q.; Zhao, W.; Li, J.; Sun, Y.; Liu, K.; Liu, B.; Zhang, N. Astragaloside IV and cycloastragenol are equally effective in inhibition of endoplasmic reticulum stress-associated TXNIP/NLRP3 inflammasome activation in the endothelium. $J$. Ethnopharmacol. 2015, 169, 210-218. [CrossRef] [PubMed]

55. Yang, S.; Zhang, R.; Xing, B.; Zhou, L.; Zhang, P.; Song, L. Astragaloside IV ameliorates preeclampsia-induced oxidative stress through the Nrf2/HO-1 pathway in a rat model. Am. J. Physiol. Endocrinol. Metab. 2020, 319, E904-E911. [CrossRef] [PubMed] 
56. Carsten, C.G., 3rd; Calton, W.C.; Johanning, J.M.; Armstrong, P.J.; Franklin, D.P.; Carey, D.J.; Elmore, J.R. Elastase is not sufficient to induce experimental abdominal aortic aneurysms. J. Vasc. Surg. 2001, 33, 1255-1262. [CrossRef] [PubMed]

57. Perez-Riverol, Y.; Csordas, A.; Bai, J.; Bernal-Llinares, M.; Hewapathirana, S.; Kundu, D.J.; Inuganti, A.; Griss, J.; Mayer, G.; Eisenacher, M.; et al. The PRIDE database and related tools and resources in 2019: Improving support for quantification data. Nucleic Acids Res. 2019, 47, D442-D450. [CrossRef] 